\title{
Resultado económico y de empleo en las organizaciones sociales de origen familiar
}

\author{
Clara Angulo Estremera \\ Ana José Bellostas Pérezgrueso \\ Francisco José López Arceiz
}

RESUMEN: La Unión Europea ha apostado por el modelo de empresa social como una herramienta para corregir los desequilibrios socioeconómicos en los países miembros. En el contexto de la economía española, las organizaciones familiares destacan debido a su fuerte presencia y apoyo. El objetivo de este trabajo es estudiar la relación entre el rendimiento económico y el nivel de empleo, como indicador de impacto social, en las organizaciones aragonesas de origen familiar, así como las diferencias en esta interacción cuando la forma lucrativa 0 no lucrativa de la estructura formada por la unidad familiar se introduce como variable moderadora. Para ello, se obtuvo una muestra de 2.931 empresas y 117 fundaciones con actividad en Aragón. Los resultados obtenidos muestran la inexistencia de relación entre la creación entre el rendimiento económico y el nivel de empleo en las organizaciones analizadas. Por otra parte, esta interacción es independiente de la forma jurídica lucrativa o no lucrativa de la organización, quedando un largo camino por recorrer en la adopción del modelo de empresa social en este tipo de organizaciones.

PALABRAS CLAVE: Rendimiento económico, Empleo, Impacto social, Empresa familiar, Fundaciones de origen familiar.

CLAVES ECONLIT: L31, L25, M10.

Cómo citar este artículo / How to cite this article: ANGULO, C. \& BELLOSTAS, A.J. \& LÓPEZ, F.J. (2017):

"Resultado económico y de empleo en las organizaciones sociales de origen familiar", CIRIEC-España, Revista de Economía Pública, Social y Cooperativa, 91, 115-147.

Correspondencia: Clara Angulo Estremera, Gdo. en Derecho y Administración y Dirección de Empresas; Ana José Bellostas Pérezgrueso, Dr. Economía Financiera y Contabilidad; Francisco José López Arceiz, Dr. Economía Financiera y Contabilidad. Departamento de Contabilidad y Finanzas de la Universidad de Zaragoza, y Departamento de Gestión de Empresas de la Universidad Pública de Navarra.

E-mail de contacto: bellosta@unizar.es. 


\section{EXPANDED ABSTRACT}

\section{Economic result and employment in social organizations of family origin}

Aim: Social enterprises are a tool to correct socioeconomic imbalances in modern welfare states. In the European context, they are responsible for the creation of economic growth, social impact and employment. These organizations can have different origins based on the cultural tradition and the evolution of each country. In the Spanish context, the majority of social enterprises are created by families, although the influence of these families in the interaction between economic result and social impact has not been studied yet. For this reason, the aim of this paper is double. Firstly, we study the relationship between the economic result and the level of employment, as indicator of social impact. Secondly, we analyze possible differences in this interaction taking into account the profit-making nature of the family structure. Both aims enable us to identify possible behaviors of social enterprise in this specific context.

Design/Methodology/Approach: These aims are analyzed through the formulation of two working hypotheses. The first hypothesis proposes a positive and significant interaction between the economic result and the level of employment, as indicator of social impact. Although the previous literature postulated a positive sign in this interaction (Albinger \& Freeman, 2000; Makni et al, 2009; Orlitzky et al, 2003; Wu, 2006; Margolis et al, 2007; Beurden \& Gössling, 2008), there are some authors who evidence no interaction (McWilliams et al. 1999 and McWilliams \& Siegel 2000) or, even, a negative pattern (Abiodun 2012). As we can observe, there is no consensus about the sign of this interaction. For this reason, we have proposed our first working hypothesis.

The second working hypothesis introduces the profit-making nature of the family structure as a moderator variable. In this paper, we identify four possible models to create social organizations from family structures. The first two models create economic and social impact from for-profit legal forms. The model 1 (traditional family enterprise) will be considered as a social enterprise model if the entity prioritizes a social aim. The model 2 (hybrid family enterprise) will be based on two entities, the family enterprise for developing economic activities and the foundation to create social impact as part of the CSR policy. The second two models are based on nonprofit legal forms. The model 3 (hybrid family foundation) is also based in the same two entities, but in this case, the enterprise will be created by the foundation as part of its economic activity. The model 4 (traditional family foundation) will promote a key social aim and the development of a market orientation in the foundation. The influence of these models is tested in our second working hypothesis. 
To test both hipotheses, we have studied a sample of Aragonese social organizations of family origin. In particular, we have 2,931 social organizations of family origin with for-profit legal form and 117 social organizations of family origin with nonprofit legal form. This sample allows us to study the interaction between the economic result and the level of employment and the moderator effect of the legal form chosen by the family. In each case, we have analyzed the accounting information related to the financial and social position of these entities. After this analysis, we have studied the correlation matrix and we have defined two dimensions related to the economic result and the level of employment by using confirmatory factor analysis (CFA). The estimation of the factor scores was studied to test the first working hypothesis. In a second step, through a multi-group analysis, we have examined the moderator effect of the profit-making nature of the family structure. This comparison is based on the Lagrange test $(\mathrm{LM})$ and it has supported the result related to the second working hypothesis.

Results/Limitations/Implications: The obtained results show a low interaction between the economic result and the level of employment created by the analyzed organizations. Moreover, differences of behavior in this relationship are not detected when the profit-making nature of the family structure is taken into account. However, this interaction is intensified in nonprofit legal forms (models 3 and 4) in some phases of the economic cycle.

Some limitations have been detected in the development of this paper. Firstly, there is a lack of consensus in the definition of the variables that measure the creation of economic result and, in particular, social impact. In this study, we have focused on the input approach through the analysis of the level of employment achieved by the organization. Secondly, we have studied a sample of Aragonese social organizations of family origin where we have detected a strong heteroscedasticity. This dispersion complicates the extrapolation of the results. Finally, the four models to create social organizations from family structures have been grouped together taking into account the profit-making nature based on the legal form. This is a limitation because other moderator variables, such as the capital intensity or the type of activity, have not been taken into consideration.

As main implication of this paper, we highlight the low level of development of the social enterprise model in the Aragonese family organizations. This result could be explained by both a contextual situation and a lack of knowledge and incentives to evolve to hybrid behaviors despite of the capacities and strengthens detected in these organizations. In any case, the social organizations of family origin with for-profit legal form (traditional family enterprise and the hybrid familiar enterprises) are the "core" of the social enterprise model, being detected a more intense tendency to create social impact.

Conclusions/originality: In this paper, we have identified four patterns of social organizations of family origin (traditional family enterprise, hybrid family enterprise, hybrid family foundation and traditional family foundation). A social activity can be developed in all these models, although our results show that there is no a positive relationship between the economic result and the level of employment achieved by these organizations. In other words, the model of behavior was really different from 
the characteristic pattern of a social enterprise in the last few years. In relation to the profit-making nature of the family structure, we can observe a moderator effect in 2010 and 2013. In particular, the models 3 (hybrid family foundation) and 4 (traditional family foundation) developed a worse interaction between the economic results and the level of employment, as indicator of social impact. These entities had to destroy employment in the downward trend of the economic cycle. Despite of this behavior, we also observe a tendency to substitute granted employment by non-granted employment in a movement which can be interpreted as a market orientation of social organizations of family origin with nonprofit legal form.

As a consequence, our results are far from the proposal of Porter \& Kramer (2011) about the creation of shared value. In these entities, there is no a complementary relationship between the economic result and the level of employment. This result does not mean that we can observe a general negative relationship as Abiodum (2012) detected. Although we have obtained some negative signs, this is no the general behavior of the studied sample. Then, our result supports the conclusion of McWilliams et al (1999) and McWilliams \& Siegel (2000) who do not detect empirical relationship between indicators of economic and social performance. In relation to the differences between the proposed models, our results do not support the conclusions of Kerlin (2006), Dorado (2006) and Thompson \& Doherti (2006). In this sense, social organizations of family origin with nonprofit legal form would not able to create a more positive relationship between the economic result and the level of employment as proxy of social impact.

In any case, this paper goes into detail about the analysis of the behavior of family organizations as social enterprises. Social enterprises are the wager of European institutions to redefine economic markets and productive processes. In our study, we evidence the development of a similar behavior from for-profit and nonprofit legal forms. Moreover, we introduce a multi-group analysis in order to evidence the differences between both types of entities.

KEYWORDS: Economic result, Social impact, Employment, Family business, Family nonprofit. 


\section{Introducción}

Las organizaciones de origen familiar desempeñan un papel dinamizador dentro de la economía de la Comunidad Autónoma de Aragón. Estas organizaciones son responsables de al menos el 68,7\% del PIB de esta Comunidad Autónoma, proporcionando empleo a más de 150.000 personas (Del Sol, 2015). Estas cifras, unidas al desarrollo de una serie de valores específicos que se transmiten a toda la organización (Asociación de Empresa Familiar de Aragón-AEFA, 2015), hacen que sea el sector donde se soporta la generación de impacto económico y social (Lattuada et al, 2015). De ahí que la potenciación de cualquier mejora en la forma de crear impacto socioeconómico necesariamente deba ir enfocada hacia las organizaciones familiares.

Desde la Unión Europea, se está promoviendo el desarrollo de nuevos modelos de comportamiento organizativo que permitan enfrentar los retos y desequilibrios socioeconómicos existentes (Estrategia 2020). La empresa social es la apuesta de las instituciones europeas para reordenar mercados y procesos productivos. Estas organizaciones se desarrollan desde distintos perfiles y formas jurídicas (Salamon y Sokolowski, 2016). Así, desde formas lucrativas y no lucrativas, las empresas sociales dan respuesta a las necesidades económicas y sociales de su entorno. Para ello, el desarrollo de actividad social será un medio de atender objetivos filantrópicos, bien como fin en sí mismo o bien como una vía complementaria a las actividades de mercado. De la misma manera, las actividades económicas podrán configurarse como objetivo preeminente o como herramienta para lograr fines sociales. En cualquiera de los casos, es necesario profundizar en el estudio del comportamiento de las organizaciones familiares como empresas sociales, dado su peso en la economía aragonesa, así como su capacidad para generar bienestar social y económico.

El objetivo de este trabajo es detectar comportamientos de empresa social entre las estructuras formadas por las organizaciones familiares aragonesas. Para ello, este estudio se desarrollará en dos etapas. En primer lugar, se estimará la posible relación entre el resultado económico y el volumen de empleo, como indicador este último de generación de impacto social. Además, en segundo lugar, se analizará cómo afecta la orientación al mercado de la organización familiar, aproximada por su forma jurídica, en la interacción anterior. Para dar respuesta a este objetivo, se cuenta con una muestra compuesta por 2.931 empresas familiares y 117 fundaciones de origen familiar pertenecientes a la Comunidad Autónoma de Aragón. Los resultados obtenidos muestran que existe una baja interacción entre el resultado económico y el volumen de empleo generado en las organizaciones analizadas. Asimismo no se detectan diferencias de comportamiento en esta relación entre distintos tipos de entidades cuando se tiene en consideración su orientación al mercado, si bien en algunos períodos esta interacción se intensifica en las entidades no lucrativas de origen familiar. 
Este trabajo está compuesto por los siguientes apartados: en primer lugar, se aborda el marco teórico del estudio, compuesto por el desarrollo y delimitación de concepto de organización familiar, orientadas y no orientadas al mercado, así como el enfoque de empresa social en el que se enmarca este estudio. Dentro del marco teórico, también se presentan las hipótesis de trabajo. Posteriormente, se describe la población y muestra utilizada, se definen las principales variables analizadas, la metodología y la estadística descriptiva. A continuación se exponen los resultados y, por último, las conclusiones del estudio.

\section{Marco teórico}

Las empresas familiares son el tipo de unidad económica más antigua (Kaplan, 2012), y posiblemente el modelo de organización orientado al mercado más enraizado en todas las economías (Hall et al, 2001, Bingham et al, 2011, Gersick y Feliu, 2014). Estas organizaciones son resultado de la iniciativa de unidades familiares, pero no son la única iniciativa posible. Así, como fruto del emprendimiento de las familias, la empresa familiar coexiste junto a fundaciones y otras estructuras de origen familiar, como expresión del perfil filantrópico de las unidades familiares (Feliu y Botero, 2015).

En estos últimos años, desde el mundo académico se ha percibido una evolución en las estrategias de las organizaciones, tanto en el mundo de los negocios como entre las entidades sin fines lucrativos. Como resultado de esta evolución surgen las empresas sociales, identificadas como modelos híbridos de comportamiento con capacidad tanto para lograr beneficios económicos como generar performance social (Pearce y Kay, 2003). Las organizaciones familiares, empresas y entidades sin fines lucrativos, destacan frente a otro tipo de entidades por rasgos tales como su capacidad de emprendimiento, la fuerte cohesión entre sus miembros, su flexibilidad para responder a cambios del entorno y por su fluidez en las interrelaciones con sus stakeholders (López-Cozar et al, 2014). Incluso autores como Bingham et al (2011) afirman que en las entidades familiares se detecta mayor implicación y capacidad de respuesta a los problemas que se manifiestan en el contexto local donde se asientan. Todas estas características evidencian que el perfil de organización familiar es un factor que potencia el desarrollo de actuaciones con impacto social y, en definitiva, la adopción de modelos de gestión guiados por objetivos socio-económicos, que serán estudiados en este trabajo bajo el prisma de la empresa social. 


\subsection{Concepto de empresa social}

El estudio de la empresa social surge en la década de los ochenta y, aunque hay numerosos trabajos científicos al respecto, existe una variedad de terminología y multitud de definiciones alternativas (Defourny y Nyssen, 2008, 2013, Kerlin, 2006, Quintao, 2007, Hulgard, 2010, Fayolle y Matlay, 2010). En el contexto norteamericano, coexisten dos escuelas de pensamiento: "The mission-oriented business" (Boschee, 2001, Emerson y Twersky, 1996, Young y Salamon, 2002, Austin et al, 2006, Yunus, 2010) y la escuela "social innovation" (Dees et al, 2001). A pesar de las diferencias que existen entre estas escuelas, ambos enfoques confluyen al identificar empresa social como aquella organización cuya estrategia está orientada tanto a objetivos sociales como económicos, con independencia de la forma jurídica adoptada (Emerson, 2006).

En la Europa Continental, el primer referente es la propuesta de la red EMES ${ }^{1}$, basada en indicadores característicos de este tipo de entidades de naturaleza empresarial, social y de gobierno (Tabla 1).

\section{Tabla 1. Características distintivas de la Empresa Social}

\begin{tabular}{|c|c|c|}
\hline Indicadores empresariales & Indicadores sociales & Indicadores de gobierno \\
\hline $\begin{array}{c}\text { Actividad continuada de mercado } \\
\text { Nivel significativo de riesgo } \\
\text { Existencia de trabajadores remunerados }\end{array}$ & $\begin{array}{c}\text { Objetivo explícito de alcanzar valor social } \\
\text { Sociedad civil como promotor } \\
\text { Limitaciones en el reparto del beneficio }\end{array}$ & $\begin{array}{c}\text { Autonomía en la gestión } \\
\text { Decisiones bajo modelo democrático } \\
\text { Participación activa de stakeholders }\end{array}$ \\
\hline
\end{tabular}

FUENTE: Elaboración propia a partir de Defourny y Nyssens (2012).

Del análisis de la propuesta de EMES, se deduce que una empresa social es una organización donde la performance económica es una herramienta para lograr fines de naturaleza social, donde los objetivos sociales prevalecen sobre el objetivo económico (Nogales, 2007; Nogales et al, 2008). Este es el punto de partida de la propuesta realizada recientemente desde el Third Sector Impact Project (TSIP) cuyo objetivo es reconceptualizar el Tercer Sector europeo y redefinir sus componentes, entre los que destaca la empresa social. Para ello, se ha utilizado como eje central el concepto de nonprofit (Salamon y Sokolowski, 2014). Esta iniciativa ha cuestionado la ubicación de la empresa social dentro del Tercer Sector europeo. Bajo esta propuesta, parte de la Economía Social europea quedaría fuera de esta definición de Tercer Sector. Esta circunstancia ha dado lugar a un intensa dialéctica entre dos líneas de pensamiento dentro de la Unión Europea (Grønbjerg, 2016 en Defourny et al, 2016, p. 1554). La primera corriente, denominada enfoque de la Economía Social defiende que entidades tales como cooperativas y mutuas deben formar parte del Tercer Sector, enfatizando su perfil de gobierno

1.- EMES es una red de investigación promovida desde distintos países europeos cuya finalidad es desarrollar un mayor nivel de conocimiento sobre la empresa social y la economía social y solidaria.http://www.emes.net/ 
democrático (Defourny et al, 2016, p. 1548). Por el contrario, la segunda corriente, conocida como enfoque nonprofit, no exige un perfil participativo en la toma de decisiones, considerando la no distribución de excedentes económicos como elemento clave para distinguir una organización del Tercer Sector (Salamon y Sokolowski, 2016, p. 1533). En consecuencia, en estos momentos, no existe unanimidad en la tipología de empresas sociales que forman parte del Tercer Sector europeo.

Ahora bien, a pesar de estas discrepancias, en el discurso académico se mantiene la esencia sobre el comportamiento que debe adoptar una empresa social (Nogales, 2017). Así, se identifica como empresa social toda aquella organización que responde a un modelo organizativo sostenible, donde las personas son más importantes que el capital (Pîrvu et al, 2009). Se trata de entidades con características propias, que se sitúan en valores, capacidades y performance a medio camino entre una empresa tradicional y una entidad sin fines lucrativos sin orientación de mercado (Pearce y Kay, 2003, Chavés y Monzón, 2012, Díaz-Foncea y Marcuello, 2012, Bellostas et al, 2016). Esta concepción es extrapolable tanto a ciertas organizaciones empresariales como a ciertas fundaciones y asociaciones (Dees, 2001, Travaglini et al, 2010, Alter, 2006, Etchart y Davis, 1999, López-Arceiz et al, 2016). En este sentido, Defourny y Nyssens (2008), Austin et al (2006), Chad (2013) y Eikenberry y Kluver (2004) pronosticaban la identificación como empresa social de todas aquellas entidades sin fines lucrativos que respondieran a comportamientos de corte empresarial. Es por ello que la empresa social ha dejado de ser un fenómeno exclusivo del ámbito empresarial, aglutinando a otro tipo de estructuras originarias del Tercer Sector que realicen actividades de mercado. La forma en que se combine la realidad de una entidad sin fines lucrativos con una gestión empresarial abre un abanico de posibilidades en las formas de hacer empresa social (Alter, 2006). Por lo tanto, atendiendo a su origen, las empresas sociales pueden ser el resultado del emprendimiento social desde la empresa, del emprendimiento económico de una entidad sin fines lucrativos, del emprendimiento socioeconómico del Estado, y de alianzas y colaboraciones entre el sector público y privado, y entre empresas y entidades sin orientación de mercado. El nexo común entre estas múltiples manifestaciones de empresa social será la búsqueda de valor social (Harding, 2004).

En definitiva, y de acuerdo con el actual concepto de empresa social propuesto por la Comisión Europea (European Commission, 2017), consideramos que una empresa social es toda aquella organización cuyo principal objetivo es generar impacto social operando desde el mercado. Se trata de un modelo de comportamiento que puede estar presente tanto en sociedades mercantiles, cooperativas o mutuas como en aquellas entidades sin fines lucrativos que de una forma u otra participan habitualmente en actividades de mercado. En todas ellas, el objetivo prioritario debe ser crear valor social, desde herramientas propias de una gestión empresarial.

\subsection{Concepto de empresa social de origen familiar}

Actualmente, la empresa social de origen familiar carece de tratamiento en la literatura especializada. Uno de los objetivos de este trabajo es aportar al desarrollo académico una delimitación de este tipo de organizaciones en el ámbito de las familias, aplicando para ello la propuesta de empresa 
social y las dimensiones de empresa familiar. Para ello, en primer lugar, es necesario acotar qué se entiende por organización de origen familiar, dado que a nivel académico aún no se ha alcanzado consenso sobre su definición (O’Boyle et al, 2010).

El origen de estas organizaciones puede encontrarse tanto en el sector lucrativo como en el Tercer Sector. Dentro del sector lucrativo, Tsang (2002), Vallejo (2005) o más recientemente Castrillón y Mares (2016) aprecian tres dimensiones comunes en los estudios comparativos sobre definiciones de empresa familiar: concentración del capital de la entidad en una familia, participación mayoritaria de miembros de la familia en la dirección estratégica del negocio e implicación de varias generaciones de una familia en la empresa. Estos son los criterios esencialmente adoptados por el Grupo Europeo de Empresas Familiares (2008), la Family Business Network (2016) y la Red de Cátedras de Empresa Familiar (RCEF, 2015) para la identificación de las empresas familiares.

No obstante, estas dimensiones son insuficientes para reflejar toda la realidad de las organizaciones familiares en la actualidad. Así, dentro del Tercer Sector, este tipo de entidades destacan por su perfil filantrópico, como expresión de la implicación y sensibilidad de una familia con su entorno, aportando un volumen considerable de recursos a la prestación de servicios sociales, educativos, sanitarios y culturales (Feliu y Botero, 2015). El ejercicio filantrópico de las familias tiene múltiples expresiones; desde la realización de donaciones, periódicas o esporádicas, hasta la planificación y ejecución de proyectos de impacto social, como máxima expresión de filantropía.

Las familias y organizaciones familiares gestionan sus proyectos sociales a través de dos estructuras formales:

- Desde formas jurídicas lucrativas. En este caso, los recursos de la entidad están destinados a lograr valor económico y valor social, y los objetivos de la organización tienen una naturaleza dual.

- Desde formas jurídicas no lucrativas fundacionales, como expresión de entidad sin fines lucrativos, creadas por las familias 0 , en su caso, las empresas familiares. En este modelo, la empresa familiar está dedicada a la generación de valor económico, mientras que la fundación está destinada a la creación de valor social.

En la literatura se identifica una fundación de origen familiar cuando una familia 0 algunos de sus miembros adoptan el papel de fundador y, además, asumen el control en el gobierno y/o en la gestión de dicha entidad (Gersick y Feliu, 2014). Respetando la naturaleza jurídica de las fundaciones, este concepto engloba algunas de las dimensiones básicas propias de la empresa familiar establecidas en España a criterio de la RECF (2015). De esta forma, la dotación fundacional será aportada mayoritariamente por una familia, o varios de sus miembros, y la dirección estratégica de la organización estará controlada por familiares, con participación mayoritaria en el patronato. La tercera dimensión exigida a una empresa familiar, voluntad de supervivencia inter generacional, será un rasgo intrínseco del objeto fundacional. Esto es, la continuidad de la fundación generación tras generación de la familia 
dependerá de que no esté vinculada a un proyecto finalista. Estas fundaciones de origen familiar convivirán en la forma de desarrollar actividad social con la empresa familiar u otras estructuras creadas por la familia (Credit Suisse, 2010, Rey-García, 2012, Rey-García y Puig-Raposo, 2013).

Por lo tanto, la incursión de las familias y organizaciones familiares en el mundo de lo social abre un abanico de modelos híbridos, en estructura y actitud, ante la creación de valor en general y más concretamente de impacto social que se sintetizan en la Tabla 2.

\section{Tabla 2. Estructura y actitud de las organizaciones familiares ante la creación de valor}

\begin{tabular}{|c|c|c|c|c|c|}
\hline $\begin{array}{l}\text { Forma } \\
\text { jurídica }\end{array}$ & Modelos & \multicolumn{3}{|c|}{ Estructura } & Actitud \\
\hline \multirow[t]{2}{*}{ Lucrativa } & \begin{tabular}{l|} 
Modelo 1. \\
Empresa \\
Familiar \\
Tradicional \\
\end{tabular} & FAMILIA & 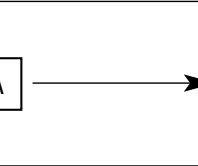 & $\begin{array}{l}\text { EMPRESA } \\
\text { FAMILIAR }\end{array}$ & $\begin{array}{l}\text { Actitud proactiva a la creación de rendi- } \\
\text { miento económico desde criterios de gestión } \\
\text { empresarial. Posibilidad de crear impacto } \\
\text { social. }\end{array}$ \\
\hline & $\begin{array}{l}\text { Modelo } 2 . \\
\text { Empresa } \\
\text { Familiar } \\
\text { Híbrida }\end{array}$ & FAMILIA & $\rightarrow \begin{array}{c}\text { FUNDACIÓN } \\
\text { FAMILIAR }\end{array}$ & $\rightarrow \begin{array}{l}\text { EMPRESA } \\
\text { FAMILIAR }\end{array}$ & $\begin{array}{l}\text { Actitud proactiva a la creación de rendi- } \\
\text { miento económico e impacto social. } \\
\text { La performance social como canal de crea- } \\
\text { ción de valor económico. }\end{array}$ \\
\hline \multirow[t]{2}{*}{ No lucrativa } & $\begin{array}{l}\text { Modelo } 3 . \\
\text { Fundación } \\
\text { Familiar } \\
\text { Híbrida }\end{array}$ & FAMILIA & $\rightarrow \begin{array}{l}\text { EMPRESA } \\
\text { FAMILIAR }\end{array}$ & $-\begin{array}{c}\text { FUNDACIÓN } \\
\text { FAMILIAR }\end{array}$ & $\begin{array}{l}\text { Actitud proactiva a la creación de rendimiento } \\
\text { económico económico e impacto social. } \\
\text { La performance económica como canal de } \\
\text { creación de impacto social. }\end{array}$ \\
\hline & \begin{tabular}{|l|} 
Modelo 4. \\
Fundación \\
Familiar \\
Tradicional
\end{tabular} & FAMILIA & & \begin{tabular}{|c|} 
FUNDACIÓN \\
FAMILIAR \\
\end{tabular} & $\begin{array}{l}\text { Actitud proactiva a la creación de impacto } \\
\text { social desde criterios de gestión de entida- } \\
\text { des sin fines lucrativos. Posibilidad de reali- } \\
\text { zar actividad de mercado. }\end{array}$ \\
\hline
\end{tabular}

FUENTE: Elaboración propia.

Puede observarse como las familias participan en la creación de resultado económico e impacto social a través del emprendimiento de negocios, desde las empresas familiares. Pero además, desde las unidades familiares también se fomenta la creación de impacto social y la participación en actividades de mercado, bien creando directamente fundaciones, o bien potenciando la creación de una fundación desde la empresa familiar. A partir de ahí, de acuerdo con Martín et al. (2017), una fundación o empresa familiar que genere actividad económica como elemento instrumental al servicio de la creación de valor social, además será catalogada como empresa social. 
Aplicando estos criterios a los modelos de organización familiar (Tabla 2), en todos los casos es posible identificar comportamientos de empresa social. La empresa familiar tradicional (modelo 1) se comportará como una empresa social en la medida en que anteponga ciertos objetivos sociales a los intereses económicos. Asimismo, en el modelo de empresa familiar híbrido (modelo 2) se apreciarán comportamientos de empresa social cuando la empresa familiar se utilice como elemento para desarrollar actividades económicas, siendo siempre objetivo primordial de este modelo la creación de impacto social sostenible desde la fundación. En el modelo 3, fundación familiar híbrida, la conducta de empresa social es más evidente, cuando la empresa familiar instrumenta la generación de impacto social a través de una fundación. Para finalizar, una fundación familiar tradicional (modelo 4) respondería a actitudes de empresa social si potencia una orientación de mercado con el fin de financiar la creación de impacto social. En consecuencia, cualquiera de los cuatro modelos puede seguir patrones de comportamiento propios o cercanos a una empresa social. De ahí, el interés de analizar la impregnación de este modelo entre las organizaciones de origen familiar.

\subsection{Hipótesis de trabajo}

En la literatura económica, se detecta un fuerte interés por estudiar las relaciones entre la creación de impacto social y el rendimiento económico en las distintas organizaciones (Ortas y Moneva, 2010). Uno de los principales problemas a los que se enfrenta el investigador es la definición de impacto social. Clifford et al (2013) definen impacto social como el conjunto de cambios intencionalmente conseguidos entre los usuarios y beneficiarios como resultado de la actividad organizacional. Dillenburg et al (2003), Polonsky y Grau $(2011,2016)$ lo definen como el impacto global que una organización genera sobre sus grupos de interés, identificando a los recursos humanos como grupo de interés crítico en las organizaciones analizadas. En particular, Boussemart et al (2017) identifican como una proxy de impacto social los niveles de empleo logrados por una organización.

A partir de esta definición, Poyatos (2015) plantea la necesidad de estudiar la relación entre rendimiento económico y varios indicadores de impacto social. Como conclusión más reseñable, este autor evidencia una interacción positiva entre el nivel de empleo, como indicador de impacto social, y los rendimientos de una organización (Poyatos, 2015, p. 231). A esta misma conclusión llegan también otros autores tales como Albinger y Freeman (2000) o Makni et al (2009). Esta relación también se confirma en estudios más amplios donde se utilizan también otros indicadores de impacto social (Orlitzky et al, 2003; Wu, 2006; Margolis et al, 2007; Beurden y Gössling, 2008). Sin embargo, otros autores no encuentran relación significativa o, incluso, obtienen una relación negativa entre ambos elementos (McWilliams et al. 1999 o McWilliams y Siegel 2000, Abiodun 2012). Por tanto, en estos momentos no se ha alcanzado consenso entre la doctrina acerca de la existencia y, en su caso, el sentido de esta relación (Brammer y Millington, 2008, Hahn y Figge, 2011, Lockett et al, 2006, Waddock y Graves, 1997). No obstante, se observa como aspecto común en muchos de estos trabajos la utilización de los niveles de empleo como indicador de impacto social. Dada esta diversidad de resultados, y la falta de análisis en el contexto de las organizaciones sociales aragonesas de origen familiar, se plantea la siguiente hipótesis de trabajo, 
$\mathrm{H}_{1}$ : Existe una relación positiva y estadísticamente significativa entre el rendimiento económico y el nivel de empleo como indicador de impacto social en las organizaciones aragonesas de origen familiar.

El rechazo de $\mathrm{H}_{1}$ implicaría que no existe una relación, o en su caso que la relación es negativa, entre ambos tipos de valor en las organizaciones aragonesas, lo que implicaría que estas entidades no serían capaces de generar rendimiento económico y empleo al unísono. Este resultado, vendría justificado por la posible existencia de variables moderadoras que podrían causar esta falta de significatividad (Gómez, 2008). Por el contrario, el no rechazo de $\mathrm{H}_{1}$ implicaría que las organizaciones familiares tienden a acercarse al paradigma de la empresa social, donde la creación de impacto social se impone como elemento crítico en la estrategia organizacional. Este signo positivo manifestaría la existencia de unas sinergias positivas ente ambos tipos de impactos de acuerdo con Gómez (2008).

Una vez analizada esta relación, es necesario tener en cuenta la orientación al mercado de la organización de acuerdo a la forma jurídica de la entidad que está detrás de los procesos de creación de impacto. Distintos autores han postulado diferencias en los procesos de creación de performance desde entidades lucrativas y organizaciones sin fines lucrativos (Kerlin, 2006). En términos generales, la intensidad de la relación entre rendimiento económico e impacto social, medido en términos de empleo, sería mayor en el sector no lucrativo dado que la entidad persigue un fin social, mantienen fuertes compromisos son sus stakeholders internos (López-Arceiz et al, 2017) y requiere de rendimiento económico para su consecución (Dorado, 2006, Thompson y Doherti, 2006). Por el contrario, en las entidades lucrativas, entre las que se encuentran las empresas familiares tradicionales, y bajo el enfoque de la empresa socialmente responsable, la actividad social y la consecución de valor social serían un elemento transitorio y voluntario, pudiendo la entidad decidir el grado de recursos económicos que destina a lo social (Galera y Borzaga, 2009). Sin embargo, a pesar de los esfuerzos teóri$\cos$, no existen trabajos que analicen empíricamente la influencia de esta variable en la relación entre rendimiento económico y nivel de empleo como indicador de impacto social en el contexto de las organizaciones analizadas, lo que lleva a plantear la siguiente hipótesis de trabajo,

$\mathrm{H}_{2}$ : El carácter lucrativo de la entidad es una variable moderadora en la relación entre la creación de rendimiento económico y nivel de empleo como indicador de impacto social en las organizaciones aragonesas de origen familiar.

El rechazo de $\mathrm{H}_{2}$ implicaría que el grado de impregnación del modelo de comportamiento como empresa social entre las organizaciones familiares aragonesas no viene condicionado por la orientación al mercado de acuerdo a la forma jurídica. En consecuencia, desde los modelos de empresa familiar tradicional y de empresa familiar híbrido es posible evolucionar hacia comportamientos propios de entidades pertenecientes al Tercer Sector. Por el contrario, el no rechazo de esta hipótesis supondría que el carácter lucrativo de la entidad influenciaría y potenciaría la interacción que pudiera existir entre rendimiento económico y nivel de creación de empleo como indicador de impacto social. 


\section{Metodología}

\subsection{Población y muestra}

A finales de 2014, en la Comunidad Autónoma de Aragón, el censo de empresas y fundaciones familiares activas es de 3.514 (Asociación de Empresa Familiar en Aragón, 2015) y 199 (Registro de Fundaciones del Gobierno de Aragón, 2015), respectivamente. Desde ambos censos se extrajo una muestra compuesta por 2.931 empresas mercantiles (83,4\% del total de población) y 117 fundaciones con orientación al mercado con domicilio y actividad en territorio aragonés ( $58,8 \%$ del total de fundaciones censadas). Con un total de 3.048 casos (el $82,1 \%$ de la población), el tamaño de la muestra es considerado estadísticamente representativo². Para desarrollar el análisis se ha atendido a la estructura formada por las distintas familias, lo que lleva a clasificar las entidades anteriores en dos categorías (tabla 2): Formas jurídicas lucrativas (modelos 1 y 2) y formas jurídicas no lucrativas (modelos 3 y 4$)$.

Para cada una de las observaciones, se obtuvo información de las Cuentas Anuales y como memorias de actividad, relativas a un periodo de 5 años (2009-2013). Para ello, se utilizó la base de datos $\mathrm{SAB}^{3}$ y se consultaron diversos registros de fundaciones (Registros del Ministerio de Sanidad, Servicios Sociales e Igualdad, del Ministerio de Educación, Cultura y Deporte y del Ministerio de Justicia, así como, del Gobierno de Aragón).

\subsection{Principales variables}

Dado el objetivo planteado en este trabajo se han definido indicadores para medir el resultado económico y el impacto social en este tipo de organizaciones. En relación al rendimiento económico, aunque no es posible encontrar un indicador universal (Medina et al, 2008), existe un cierto consenso en la literatura en identificar este concepto con la rentabilidad económica generada (López-Arceiz et al., 2016a, López-Arceiz et al., 2016b). Burns et al. (2008), Gheorghe (2013) o Herciu et al. (2014) proponen la descomposición del ratio de rentabilidad en margen y rotación (sistema Du Pont), dado que a partir de ambos indicadores se puede obtener información sobre la capacidad de la entidad para generar rendimiento por unidad de venta (margen), así como su habilidad para renovar su activo (rotación). Esta doble dimensionalidad hace que el rendimiento económico sea una variable no observable

2.- Para calcular la representatividad se ha asumido una distribución Bernoulli ( $\mu=p ; \mu=p(1-p))$, así como niveles de significatividad y error máximo del $5 \%$. A partir de la siguiente expresión $\left(\left|p_{n}-p\right| \leq e^{\max }\right) \geq 0,95$ se obtiene que el tamaño muestral mínimo para población finita es 346,30.

3.- Sistema de Análisis de Balances Ibéricos. 
directamente que se debe aproximar a través de distintos indicadores vinculados a margen y rotación (Chang et al, 2014). En concreto, en este trabajo se ha hecho uso de cuatro ratios: a) Margen de explotación (Resultado de la explotación/Ingreso de la explotación), b) Margen antes de impuestos (Beneficio antes de impuestos/Ingreso de la explotación), c) Margen neto (Beneficio neto/Ingresos de la explotación) y, d) Rotación (Ingreso de la explotación/ Activo total).

La medición del impacto social adolece de problemas similares. En este sentido, López-Arceiz et al (2016) ponen de manifiesto como el impacto social no puede ser medido directamente, pudiendo ser aproximado desde distintos enfoques ${ }^{4}$. En este estudio y, dado el objetivo de trabajo, se ha utilizado el volumen de empleo alcanzado como proxy del nivel de impacto social creado por una organización (enfoque de los imputs). Cuganesan (2006) 0, más recientemente, Mano $(2014,2015) 0$ Retolaza y San-José (2016) son algunos de los trabajos que han apostado por esta variable como proxy de impacto social. Esta misma propuesta es asumida por la normativa contable (Resoluciones de 26 de Marzo de 2013 (BOE n. 85, de 9 de abril de 2013, y BOE n. 86 de 10 de abril de 2013) donde se asocia la medición de actividad social a los recursos humanos empleados por la organización. También este indicador es utilizado por distintos organizaciones profesionales (CEPES, 2011; Deloitte, 2015) y forma parte de la batería de indicadores para medir impacto social propuestos por la Comisión Europea $(2011,2014)$. Además, las organizaciones familiares destacan en términos generales por su capacidad para crear empleo y favorecer un uso eficiente de sus recursos humanos (Donckels, 1998, Sharma, 2004, Ibrahim et al, 2001).

A partir de estos análisis previos, en este trabajo, se han utilizado tres indicadores de nivel de empleo como indicador de impacto social. El primer indicador, denominado empleabilidad global, relaciona el nivel de inversión y financiación por unidad monetaria de personal. Este ratio permite medir el nivel de empleo logrado aislando el tamaño de la organización (Martin et al, 2017). El segundo indicador sobre empleabilidad subvencionada mide la utilización de subvenciones como vía para la generación de empleo. Para ello se han tomado en consideración las subvenciones dirigidas a la creación de empleo vinculado a la actividad de explotación de la organización (Santos, 2015). Finalmente, se ha tomado en consideración como indicador de productividad el ratio de empleabilidad no subvencionada, que relaciona el nivel de ingreso conseguido y los trabajadores empleados (López-Arceiz et al, 2016).

La utilización de estos indicadores permite obtener variables proxy de resultado económico e impacto social de una organización; siendo posible a través de ellas estudiar las hipótesis propuestas. Además, se ha introducido la forma lucrativa (modelos 1 y 2) y la forma no lucrativa (modelos 3 y 4) como variable dummy con objeto de testar la segunda hipótesis. Finalmente, se ha considerado el sector de actividad como variable de control.

4.- Los indicadores utilizados dependerán del enfoque de medición asumido. En la actualidad, conviven en la literatura tres enfoques: a) Medición desde los outputs (Guzmán y Trujillo, 2008; SEKN, 2006), b) Medición desde los procesos (Mair y Marti, 2006, p 37, Anderson y Dees, 2002, Peredo y Mclean, 2006) y c) Medición desde los imputs (Austin et al, 2006, Sud et al, 2009, Felicio et al, 2013). 


\subsection{Metodología}

Para dar respuesta al objetivo de este trabajo se han seguido los siguientes pasos. En primer lugar, se ha llevado a cabo un análisis estadístico descriptivo de los indicadores obtenidos a partir de las Cuentas Anuales y memorias de actividad. El objetivo de este análisis es conocer la situación de las organizaciones familiares en Aragón atendiendo a su forma lucrativa o no lucrativa.

A continuación, a partir de la tipificación de las distintas masas patrimoniales, se han construido los ratios de rendimiento económico y nivel de empleo presentados en el apartado anterior. Una vez obtenidos estos ratios, se ha analizado la matriz de correlaciones característica. Con la finalidad de detectar las dimensiones relacionadas con el rendimiento económico y el nivel de empleo logrado se ha planteado el uso de análisis factorial confirmatorio (AFC). Para efectuar este análisis se sigue el enfoque propuesto por Hayduk y Littvay (2012), asumiendo que cuando sólo existe un único indicador su error de medida es igual a cero. La validez convergente del modelo AFC se analizó a través de tres coeficientes: La varianza media extraída (AVE), el coeficiente omega (CRC) y el alfa de Cronbach. La validez discriminante se evaluó a partir de la matriz de correlaciones de los constructos estimados (Bagozzi, 2010).

La estimación del modelo AFC permitió calcular las puntuaciones factoriales que se utilizaron posteriormente para contrastar las hipótesis de partida. Finalmente, el análisis de correlaciones mediante un enfoque multigrupo (Bentler, 2006), tanto para organizaciones familiares con forma jurídica lucrativa (modelos 1 y 2) como no lucrativa (modelos 3 y 4), permitió concluir acerca de la relación entre resultado económico y nivel de empleo. Este análisis se replicó para la variable de control sector de actividad. La comparación entre las distintas correlaciones se realizó través del test de Lagrange (LM). El software utilizado fue SPSS v.22 y EQS 6.2.

\subsection{Estadística descriptiva}

En la tabla 3, se muestran las distintas masas patrimoniales que caracterizan a las entidades analizadas. Las variables que definen el tamaño de la entidad muestran diferencias entre las formas lucrativas correspondientes a los modelos 1 y 2 y las no lucrativas (modelos 3 y 4). A nivel de activo [Año 2009 AT: 4.216.101,880 euros; Año 2013 AT: 4.561.186,490 euros] [Año 2009 AT: 1.767.567,667 euros; Año 2013; AT: 960.867,086 euros] y pasivo [PT: 2217225 euros; Año 2013; PT: 2245572 euros; Año 2009 PT: 428.544,56 euros; Año 2013 PT: 588.123,726 euros] se observan tamaños superiores en las entidades con forma jurídica lucrativa. Este patrón también se mantiene al analizar ingresos y beneficio neto ${ }^{5}$. Desde el punto de vista de los gastos de personal, estos se mantienen constantes a

5.- A nivel de cuenta de resultados, es posible apreciar cómo el INCN es mayor en las formas lucrativas que en las no lucrativas, observándose además trayectorias distintas durante el período analizado. Respecto a la media del Beneficio Neto, observamos que es mayor en las formas lucrativas. Así, se aprecia una fuerte inestabilidad [Año 2009 BN: 112.448,137 euros; Año 2013 BN: 76.929,117 euros], que no sería tan acusada en las formas no lucrativas, quienes conseguirían mantener sus valores iniciales en esta partida [Año 2009 BN: 24.116, 756 euros; Año 2013 BN: 22.264,209 euros]. 
lo largo de los años analizados Además no existe gran diferencia entre el montante de gastos de personal atribuidos a ambos tipos de entidades. En relación a las subvenciones se observa como en términos generales éstas son mucho mayores en las formas no lucrativas que en las lucrativas [Año 2009: 3.943,579 euros; Año 2013: 3.239,766 euros; Año 2009: 281.934 euros; Año 2013: 691.719,997 euros]. Finalmente, en cuanto al número de trabajadores, la media obtenida es también superior en las fundaciones de origen familiar. No obstante, estas cifras deben ser tomadas con una cierta cautela, ya que se han observado fuertes desviaciones típicas en la muestra. Por este motivo, se decidió tipificar las variables de cara a contrastar las hipótesis planteadas.

\section{Tabla 3. Masas patrimoniales para formas jurídicas lucrativas (FL) y no lucrativas (FNL)}

\begin{tabular}{|c|c|c|c|c|c|c|c|c|c|c|c|c|c|c|c|c|c|c|c|c|}
\hline & \multicolumn{4}{|c|}{2009} & \multicolumn{4}{|c|}{2010} & \multicolumn{4}{|c|}{2011} & \multicolumn{4}{|c|}{2012} & \multicolumn{4}{|c|}{2013} \\
\hline & \multicolumn{2}{|r|}{$\mathrm{FL}$} & \multicolumn{2}{|c|}{ FNL } & \multicolumn{2}{|c|}{$\mathrm{FL}$} & \multicolumn{2}{|c|}{ FNL } & \multicolumn{2}{|r|}{$\mathrm{FL}$} & \multicolumn{2}{|c|}{ FNL } & \multicolumn{2}{|c|}{$\mathrm{FL}$} & \multicolumn{2}{|c|}{ FNL } & \multicolumn{2}{|c|}{$\mathrm{FL}$} & \multicolumn{2}{|c|}{ FNL } \\
\hline & Media & Std.Dev & Media & Std.Dev & Media & Std.Dev & Media & Std.Dev & Media & Std.Dev & Media & Std.Dev & Media & Std.Dev & Media & Std.Dev & Media & Std. Dev & Media & Std. Dev \\
\hline AT & 4216,101 & 27206,047 & 1767,567 & 4454,167 & 4515,759 & 34477,772 & 1766,351 & 4628,880 & 4664,635 & 39778,722 & 1504,571 & 3213,815 & 4618,217 & 39751,258 & 1781,285 & 4596,403 & 4566,186 & 40241,247 & 960,867 & 2031,494 \\
\hline PT & 2217,225 & 10965,957 & 428,544 & 1031,311 & 2398,998 & 15411,254 & 674,837 & 1421,659 & 2459,674 & 18883,027 & 678,937 & 1394,810 & 2368,594 & 17584,532 & 721,446 & 1484,957 & 2245,572 & 16519,896 & 588,123 & 1412,141 \\
\hline INCN & |370, & 12285,449 & 735,903 & 2069,823 & 3940,453 & 17308,992 & 675,444 & 2088,813 & 4221,606 & 20481,681 & 536,168 & 1500,765 & 4149, 4242 & 19795,366 & 644,411 & 1946,554 & 4118,838 & 21146,154 & 665,464 & 1442,659 \\
\hline R.Ex & 171,004 & 918,600 & 16,671 & 134,601 & 183,364 & 2495,964 & 2,896 & 103,914 & 167,689 & 3112,635 & 0,927 & 105,763 & 129,824 & 2697,009 & 10,891 & 121,562 & 134,139 & 2980,574 & 21,349 & 104,796 \\
\hline BAT & 144,880 & 1186,188 & 26,785 & 184,985 & 152,803 & 2636,652 & 5,468 & 101,806 & 118,011 & 3322,206 & 2,249 & 102,435 & 91,630 & 2723,126 & 19,785 & 213,610 & 107,727 & 3088,221 & 22,392 & 105,209 \\
\hline Is & 32,428 & 389,137 & 3,348 & 19,522 & 38,652 & 778,406 & 0,983 & 11,559 & 30,976 & 914,729 & 0,662 & 11,777 & 31,205 & 791,632 & 4,239 & 41,945 & 30,798 & 911,321 & 0,127 & 0,771 \\
\hline BN & 112,448 & 979,556 & 24,116 & 169,232 & 114,084 & 1868,421 & 5,746 & 97,027 & 87,035 & 2414,049 & 3,241 & 98,884 & 60,425 & 1974,990 & 15,720 & 173,416 & 76,929 & 2185,840 & 22,264 & 105,192 \\
\hline GP & 681,513 & 2132,233 & 551,677 & 1177,135 & 693,715 & 2210,861 & 543,756 & 1176,739 & 714,336 & 2289,675 & 438,187 & 1005,084 & 696,088 & 2272,313 & 484,126 & 1091,842 & 682,281 & 2386,598 & 554,266 & 1334,359 \\
\hline SE & 3,943 & 90,929 & 281,934 & 1116,202 & 5,615 & 123,527 & 392,648 & 1071,871 & 4,142 & 96,926 & 333,105 & 1032,521 & 3,190 & 93,843 & 243,841 & 477,042 & 3,239 & 87,056 & 691,719 & 2568,116 \\
\hline SC & 19,605 & 148,233 & 379,844 & 1022,686 & 20,570 & 147,756 & 369,578 & 1579,206 & 20,660 & 139,352 & 480,538 & 1827,155 & 19,895 & 130,178 & 474,827 & 2038,068 & 18,772 & 123,232 & 2017,167 & 10422,223 \\
\hline Nitiab & 22,950 & 66,344 & 27,807 & 49,849 & 23,095 & 71,344 & 32,217 & 48,815 & 23,244 & 70,355 & 25,246 & 43,039 & 22,337 & 65,813 & 21,475 & 40,114 & 21,931 & 67,143 & 32,177 & 91,657 \\
\hline
\end{tabular}

Nota: Las distintas masas patrimoniales se muestran en miles de euros. El número de trabajadores se ha medido en unidades de trabajador equivalente.

FL: Formas lucrativas (modelos 1 y 2), $\quad$ FNL: Formas no lucrativas (modelos 3 y 4)

AT: Activo Total

BN: Beneficio Neto

PT: Pasivo Total

GP: Gastos de Personal

INCN: Importe Neto de la Cifra de Negocios

SE: Subvenciones de Explotación

R. Ex.: Resultado de explotación

SC: Subvenciones de Capital

BAT: Beneficio antes de impuestos

N.trab:: Número de trabajadores

IS: Impuesto de Sociedades 
Una vez analizados los momentos de primer orden, la tabla 4 muestra la matriz de correlaciones para los distintos indicadores de rendimiento económico y nivel de empleo 6 .

\section{Tabla 4. Correlaciones para formas jurídicas lucrativas (FL) y no lucrativas (FNL)}

\begin{tabular}{|c|c|c|c|c|c|c|c|c|c|}
\hline & FL & EG1 & EG2 & ES & ENS & M1 & M2 & M3 & Rot \\
\hline EG1 & Activo/Gastos de Personal & 1,000 & & & & & & & \\
\hline EG2 & Pasivo/Gastos de Personal & $0,765^{* *}$ & 1,000 & & & & & & \\
\hline ES & Subvenciones/ $\mathrm{N}^{\circ}$ trabajadores & 0,000 & $-0,004$ & 1,000 & & & & & \\
\hline ENS & INCN/N trabajadores & $-0,019^{*}$ & $-0,29^{* *}$ & $0,224^{* *}$ & 1,000 & & & & \\
\hline M1 & Rdo. Exp/ Ing. Expl & 0,000 & 0,000 & $-0,017^{\star}$ & 0,000 & 1,000 & & & \\
\hline M2 & BAT/Ing. Expl & 0,000 & $-0,002$ & $-0,024^{* *}$ & 0,000 & $0,950^{\star *}$ & 1,000 & & \\
\hline M3 & BN/ Ing. Expl & 0,000 & $-0,002$ & $0,025^{\star *}$ & 0,000 & $0,939^{* *}$ & $0,997^{\star *}$ & 1,000 & \\
\hline \multirow[t]{2}{*}{ Rot } & Ing.Exp/ Activo & 0,000 & 0,000 & $-0,001$ & 0,001 & 0,000 & 0,000 & 0,000 & 1,000 \\
\hline & FNL & EG1 & EG2 & ES & ENS & M1 & M2 & M3 & Rot \\
\hline EG1 & Activo/Gastos de Personal & 1,000 & & & & & & & \\
\hline EG2 & Pasivo/Gastos de Personal & $0,989^{\star *}$ & 1,000 & & & & & & \\
\hline ES & Subvenciones/ $\mathrm{N}^{\circ}$ trabajadores & 0,001 & 0,003 & 1,000 & & & & & \\
\hline ENS & INCN/N ${ }^{\circ}$ trabajadores & $-0,001$ & $-0,003$ & $-0,998^{\star *}$ & 1,000 & & & & \\
\hline M1 & Rdo. Exp/ Ing. Expl & $-0,093^{*}$ & $-0,104^{*}$ & 0,032 & $-0,030$ & 1,000 & & & \\
\hline M2 & BAT/Ing. Expl & $-0,089^{*}$ & $-0,098^{*}$ & 0,032 & $-0,029$ & $0,945^{\star *}$ & 1,000 & & \\
\hline M3 & BN/ Ing. Expl & $-0,070$ & $-0,080$ & 0,033 & $-0,030$ & $0,897^{\star \star}$ & $0,978^{\star *}$ & 1,000 & \\
\hline Rot & Ing.Exp/ Activo & 0,007 & 0,007 & $-0,054$ & 0,054 & $-0,048$ & $-0,048$ & $-0,043$ & 1,000 \\
\hline
\end{tabular}

${ }^{* * *}$ pvalor $<0.01$; **pvalor<0.05; "pvalor<0.10; EM: Empresa familiar; FF: Fundación de origen familiar

En la tabla anterior, se observa como ambos tipos de organización muestran una correlación significativa entre los dos indicadores de empleabilidad global propuestos (Corr FL: 0,765; Corr FNL: 0,989). Estos valores evidencian la existencia de una dimensión latente relacionada con el volumen de empleabilidad global. Asimismo se observa cómo no existe una correlación fuerte entre los indicadores de empleabilidad subvencionada y no subvencionada. Por tanto, se propone la medición del nivel de empleo a partir de un constructo, empleabilidad global, y dos indicadores, empleabilidad subvencionada y no subvencionada.

Por otro lado, se observa cómo, con independencia de su forma lucrativa, las organizaciones sociales aragonesas de origen familiar muestran una fuerte correlación entre los indicadores de margen económico (Corr FL: [0,939-0,997]; Corr FNL: [0,897-0,978]). Por el contrario no se observan correlaciones fuertes relacionadas con el ratio de rotación. En consecuencia, los datos confirman la medición del rendimiento económico a partir de un constructo, margen, y un indicador, rotación.

6.- La tabla 4 no recoge media y desviación típica de las variables tipificadas al ser estas cero y uno respectivamente. 


\section{Resultados}

La tabla 5 muestra los resultados del modelo AFC tanto en términos de bondad del ajuste como en relación a las cargas factoriales estimadas.

\section{Tabla 5. Modelo de medida de los parámetros y de los coeficientes de fiabilidad}

\begin{tabular}{|c|c|c|c|c|c|c|c|c|c|}
\hline & & Estimador & Std.Dev. & $p$-valor & R2 & $\mathrm{p}$-valor & AVE & CRC & Alfa \\
\hline $\begin{array}{l}\text { Nivel } \\
\text { Empl }\end{array}$ & $\begin{array}{l}\text { de empleo-Impacto social } \\
\text { eabilidad Global }\end{array}$ & & & & & & & & \\
\hline EG1 & Activo/Gastos de Personal & 0,568 & 0,194 & $* * *$ & 0,585 & *** & 0,793 & 0,784 & 0,845 \\
\hline EG2 & Pasivo/Gastos de Personal & 1,000 & - & - & 1,000 & - & & & \\
\hline Empl & eabilidad Subvencionada & & & & & & & & \\
\hline ES & Subvenciones $/ \mathrm{N}^{\circ}$ trabajadores & 1,000 & - & - & 1,000 & - & 1,000 & 1,000 & 1,000 \\
\hline Empl & eabilidad No Subvencionada & & & & & & & & \\
\hline ENS & INCN/No ${ }^{\circ}$ trabajadores & 1,000 & - & - & 1,000 & - & 1,000 & 1,000 & 1,000 \\
\hline $\begin{array}{l}\text { Rend } \\
\text { Rotac }\end{array}$ & $\begin{array}{l}\text { imiento Económico } \\
\text { ión }\end{array}$ & & & & & & & & \\
\hline Rot & Ing.Exp/ Activo & 1,000 & - & - & 1,000 & - & 1,000 & 1,000 & 1,000 \\
\hline Marg & & & & & & & & & \\
\hline M1 & Rdo. Exp/ Ing. Expl & 1,000 & - & - & 1,000 & - & 0,961 & 0,999 & 0,987 \\
\hline M2 & BAT/Ing. Expl & 0,999 & 0,090 & *** & 0,896 & *** & & & \\
\hline M3 & BN/Ing. Expl & 0,999 & 0,113 & *** & 0,987 & $* \star \star$ & & & \\
\hline
\end{tabular}

C2[2] $=1,680$, pvalor: 0,432; RMSEA $=0,000$, SRMR $=0,001, \mathrm{MFI}=0,999$

${ }^{* * *}$ p-valor $<0,01 ;{ }^{* *} \mathrm{p}$-valor $<0,05 ;{ }^{*} \mathrm{p}$-valor $<0,10$

En relación al ajuste global del modelo es posible apreciar un ajuste razonable ( $\chi^{2}[2]: 1.680, p$-valor: 0,432; RMSEA: 0,000, SRMR: 0,001, MFI: 0,999), lo que permite valorar los resultados obtenidos. En relación a los ajustes individuales se observa como los coeficientes de fiabilidad de las variables latentes superan los valores de referencia (Bagozzi, 2010). [Empleabilidad Global: 0,793 (AVE) y 0,784 (CRC); Margen: 0,961 (AVE) y 0,999 (CRC)], lo que mostraría validez convergente en el modelo analizado. Estos valores se ven corroborados por el indicador alfa de Cronbach, el cual es superior a 0,8 (George y Mallery, 2003). Estos resultados permiten estimar las puntuaciones factoriales de cara a analizar la correlación entre rendimiento económico y nivel de empleo como indicador de impacto social. 
La tabla 6 muestra la matriz de correlaciones entre los indicadores de rendimiento económico y los indicadores de nivel de empleo.

\section{Tabla 6. Relación entre valor económico y valor social}

\begin{tabular}{|lc|ccccc|}
\hline & & EG & ES & ENS & Rot & M \\
\hline Nivel de empleo-Impacto social & EG & 1,000 & & & & \\
& ES & 0,000 & 1,000 & & & \\
& ENS & $-0,019^{\star}$ & $-0,775^{\star *}$ & 1,000 & & \\
\hline Rendimiento económico & Rot & 0,000 & 0,000 & 0,001 & 1,000 & \\
& M & $-0,004$ & 0,000 & 0,000 & 0,000 & 1,000 \\
\hline
\end{tabular}

${ }^{* * *}$ p-valor $<0,01 ;{ }^{* *} p$-valor $<0,05 ;{ }^{*}$-valor $<0,10$

En la tabla anterior, puede evidenciarse validez discriminante en la medición de los distintos aspectos de ambos elementos al ser las correlaciones próximas a cero o negativas (Correlación máxima rendimiento económico: 0,000; Correlación máxima nivel de empleo: 0.000). Al analizar la interacción entre rendimiento económico y nivel de empleo para el conjunto de organizaciones familiares se observa como no existe relación entre los indicadores que definen rendimiento económico y aquellos que caracterizan el nivel de empleo logrado al no ser significativos ninguno de los coeficientes obtenidos (pvalor $>0.100$ ). Este resultado lleva a rechazar la $\mathrm{H}_{1}$, al no existir una relación positiva y estadísticamente significativa entre el rendimiento económico y el nivel de empleo como indicador de impacto social en las organizaciones aragonesas de origen familiar. No obstante, este resultado podría verse alterado por el momento en el que se desarrolla la actividad, la actividad propiamente dicha y la forma lucrativa de la organización. Por este motivo, en los Anexos I y II se ha estimado la matriz de correlaciones teniendo en cuenta las distintas variables.

En relación a la $\mathrm{H}_{1}$ se obtienen resultados poco concluyentes y con grandes variaciones en función del período considerado. Así, para el año 2009 se observa una relación positiva entre empleabilidad no subvencionada y margen económico en las organizaciones bajo formas lucrativas (Margen: 0.037 ; p-valor $<0.05$ ). Es decir, las entidades con mayor actividad económica contrataban a más personal sin subvencionar. Por el contrario, en el caso de las formas no lucrativas, el nivel de rendimiento económico no llega a afectar el nivel de empleo generado. En el año 2010, se observa el resultado opuesto. Así, en las organizaciones bajo formas lucrativas, el ratio de rotación afecta de forma negativa a la empleabilidad subvencionada (Rotación: $-0,080$; $p$-valor $<0,01$ ). Esto implicaría que las entidades que contaron con más rotación en sus activos contaron con una empleabilidad subvencionada menor. Por su parte, las organizaciones bajo formas no lucrativas, muestran una relación negativa entre margen y empleabilidad global lo que dificultaría los procesos de creación simultánea de impacto en estas organizaciones (Margen: -0.347 ; p-valor $<0,01$ ). En otras palabras, en este período, las entidades sin fines lucrativos que generaron mayor margen, lo hicieron en detrimento del volumen de con- 
tratación. Este resultado también se pone de relieve cuando se introduce el sector de actividad. Así, en 2009, el sector vinculado a sanidad y servicios sociales mantiene los márgenes pero a costa de reducir el volumen de empleo (Margen: -0.320; $p$-valor $<0,10$ ). Esta situación se acentúa en el año 2010, siendo las correlaciones incluso más negativas en este sector (Rotación: $-0.358 ; p$-valor $<0,05$ ). Finalmente, en los restantes años, los resultados obtenidos muestran falta de significatividad en la relación (Año 2011) o impiden concluir dado que se obtienen resultados diversos en función del indicador utilizado (Años 2012 y 2013) ${ }^{7}$.

La $\mathrm{H}_{2}$ introduce el efecto moderador de la forma lucrativa o no lucrativa de la estructura creada por la unidad familiar. Puede observarse como las formas no lucrativas introducen un comportamiento diferente, caracterizado por intensificar el signo de la relación. No obstante al igual que ocurría en el caso anterior, se observan algunos períodos en los que este resultado no se produce, siendo la forma lucrativa o no lucrativa una variable incapaz de explicar diferencias entre los distintos parámetros.

La tabla 7 muestra un resumen para cada una de las hipótesis en función del período analizado.

\section{Tabla 7. Resumen de resultados}

\begin{tabular}{|l|cc|cc|}
\hline Año & \multicolumn{2}{|c|}{$\begin{array}{l}\mathbf{H}_{\mathbf{1}} \text {. Relación Rendimiento económico } \\
\text { y nivel de empleo-impacto social }\end{array}$} & \multicolumn{2}{c|}{ H2. Moderación: Forma lucrativa } \\
\hline 2009 & No rechazo & Relación positiva & Rechazo & - \\
2010 & Rechazo & Relación negativa & No rechazo & Mayor intensidad FLN \\
2011 & Rechazo & - & Rechazo & - \\
2012 & Inconclusa & - & Rechazo & - \\
2013 & Inconclusa & - & No rechazo & Mayor intensidad FNL \\
\hline
\end{tabular}

En definitiva, con relación a la $\mathrm{H}_{1}$ no es posible alcanzar una conclusión al observarse sentidos diferentes en la relación entre los distintos de períodos, así como incluso dentro del mismo período analizado. Dichos resultados, se ven además afectados por el sector de actividad. No obstante, en la mayor parte de los años se observa una relación negativa o inexistente. En relación a la $\mathrm{H}_{2}$ es posible observar una falta de influencia en términos generales de la forma lucrativa de la entidad, con las excepciones de los años 2010 y 2013, donde la interacción entre rendimiento económico y nivel de empleo como proxy de impacto social se intensificaría en las organizaciones bajo formas no lucrativas.

7.- En el año 2012, se observa como las formas no lucrativas poseen una mayor capacidad para crear impacto social en forma de empleo no subvencionado. No obstante, llama la atención la destrucción de empleo subvencionado. Algo similar ocurre en 2013, donde las formas no lucrativas disminuyen la dependencia del empleo subvencionado (Margen: -0.253; $p$-valor <0.05) y ganan cuotas de empleo no subvencionado. Es decir, las entidades con mayor margen tienden a mercantilizarse en sus modalidades de contratación. Estos resultados impide concluir acerca de H1 dado que la interacción entre rendimiento económico e impacto social dependerá de los indicadores utilizados.. 


\section{Conclusiones}

Las organizaciones familiares constituyen uno de los pilares básicos en el desarrollo socioeconómico español. Su peso en el PIB autonómico y nacional, en el mercado laboral y su arraigo en la sociedad son motivos que justifican el estudio de sus capacidades tanto desde el plano económico como social. Estas entidades pueden adoptar diversas estructuras que permiten su consideración como posibles entidades del Tercer Sector. En este trabajo se han identificado cuatro patrones de organizaciones familiares (empresa familiar, empresa familiar híbrida, fundación familiar híbrida y fundación familiar tradicional) desde los que se podría ejercer potencialmente una actividad social de forma prevalente. El objetivo de este trabajo ha sido analizar el grado de profundización del modelo de comportamiento de empresa social en cada uno de los cuatro patrones identificados entre las organizaciones familiares. Para ello, se ha evaluado la interacción entre rendimiento económico y nivel de empleo como indicador de impacto social. Además, se ha estudiado el rol de cada uno de los patrones, identificados de acuerdo a la forma jurídica lucrativa o no lucrativa, como variable moderadora en esta interacción.

Los resultados obtenidos ponen de manifiesto cómo durante la mayoría de los años analizados no existió una relación positiva entre los niveles de rendimiento económico y el nivel de empleo alcanzado por las organizaciones familiares aragonesas. En otras palabras, en esos años el modelo de comportamiento de las organizaciones familiares en su conjunto distó notablemente del patrón esperado para una empresa social. Respecto a la influencia de la forma jurídica lucrativa puede observarse como en los años 2010 y 2013 esta variable tuvo un efecto moderador. En concreto, las realidades más próximas al Tercer Sector, modelo de fundación familiar híbrida y fundación familiar tradicional, desarrollaron una peor interacción entre rendimiento económico y nivel de empleo como indicador de social, viéndose obligadas a destruir empleo en los momentos bajistas del ciclo económico. Ahora bien, este resultado debe ser puesto en el contexto del período de crisis atravesado por la economía española, que habría incidido de forma notable en la intensidad y signo de la interrelación. A pesar de ello, con la finalidad de proteger unos ciertos niveles de empleo se ha observado una sustitución de empleo subvencionado por empleo no subvencionado en lo que podría interpretarse como una orientación al mercado por parte de las entidades sin fines lucrativos de origen familiar.

Estos resultados se alejan de las propuesta de Porter y Kramer (2011) sobre la creación de valor compartido puesto que tal y como se ha expuesto no se ha encontrado en este tipo de entidades de corte familiar una relación de complementariedad entre la creación de rendimiento económico y nivel de empleo como indicador de impacto social. Por otra parte, los resultados obtenidos también se alejan de los alcanzados por Abiodum (2012) quien detectaba una relación negativa entre ambas variables. Aunque en el presente trabajo se ha obtenido alguna relación negativa, no es posible afirmar que 
este signo se mantenga para todo el período analizado. Por tanto, nuestro resultado estaría en línea con McWilliams et al (1999) o McWilliams y Siegel (2000), quienes no encuentran relación empírica entre indicadores de rendimiento económico e impacto social. En consecuencia, las organizaciones aragonesas de origen familiar crean valor económico e impacto social de forma no armónica, sin existir actualmente una relación sostenida entre ellos (Pirson, 2011). Esta falta de interacción podría derivarse de la implantación de una estrategia multiobjetivo, lo que haría que en el largo plazo estas organizaciones redefinieran sus procesos y estrategias en el marco de los principios y valores de la economía social. En relación a las diferencias en esta interacción entre los distintos patrones de comportamiento el resultado obtenido para las organizaciones familiares aragonesas contraviene los resultados obtenidos en marcos más generales por autores como Kerlin (2006), Dorado (2006) o Thompson y Doherti (2006). De esta forma, en el contexto analizado, los patrones pertenecientes a formas jurídicas no lucrativas no serían capaces de generar una relación más positiva entre rendimiento económico y nivel de empleo como proxy de impacto social.

Como principales implicaciones obtenidas en este trabajo, destaca un bajo nivel de profundización del modelo de comportamiento de empresa social en las organizaciones familiares aragonesas. Este resultado podría ser debido a una situación meramente contextual unida a una ausencia de conocimiento y falta de incentivos para evolucionar hacia comportamientos híbridos, a pesar de las fortalezas y capacidades detectadas en estas organizaciones. Además, las formas lucrativas (empresa familiar tradicional y empresa familiar hibrida) se ubicarían como "core" en los modelos híbridos entre las organizaciones familiares, detectándose una tendencia más favorable a la creación de impacto social.

Finalmente, es necesario poner de relieve algunas limitaciones detectadas en el desarrollo de este estudio. En primer lugar, es necesario señalar la falta de consenso a la hora de medir las variables que influyen en la creación de rendimiento económico y, en particular, de impacto social. En este estudio se ha enfocado la medición de impacto desde los imputs utilizados, analizando el volumen de empleo alcanzado por la organización. Este enfoque es un punto de vista específico que podría ser completado con indicadores pertenecientes a otros enfoques. Asimismo, en la estimación de los distintos impactos no se ha tenido en consideración el posible efecto de la normativa fiscal. En segundo lugar, se ha trabajado con una muestra limitada a la Comunidad Autónoma de Aragón, detectándose una fuerte heterocedasticidad, lo que dificultaría la extrapolación de los resultados a otras poblaciones. Finalmente, los cuatro patrones percibidos han sido agrupados en el análisis empírico en dos categorías atendiendo a la forma jurídica final de la estructura creada por la unidad familiar, lo que constituye una limitación al no haberse hecho uso de otras variables moderadoras tales como la intensidad capital-trabajo o el tipo de actividad. Por estos motivos, este trabajo queda abierto a futuras investigaciones que superen las limitaciones señaladas. 


\section{Referencias bibliográficas}

ABIODUN, B.Y. (2012): "The impact of Corporate Social Responsibility on firms profitability in Nigeria", European Journal of Economics, Finance and Administrative Sciences, 45, 39-50.

ALBINGER, H.S. \& FREEMAN, S.J. (2000): "Corporate social performance and attractiveness as an employer to different job seeking populations", Journal of Business Ethics, 28(3), 243-253.

ALTER, S.K. (2006): "Social enterprise models and their mission and money relationships". En NICHOLLS, A. (ed.): Social entrepreneurship: New paradigms of sustainable social change, Oxford University Press, Oxford.

ANDERSON, B.B. \& DEES, J.G. (2002): Developing viable earned income strategies in strategic tools for social entrepreneurs: Enhancing the performance of your enterprising nonprofit, John Wiley \& Sons Inc, Nueva York.

AEFA (2015): http://www.aefaragon.es/ [Consulta realizada el día 12 de Febrero de 2016]

AUSTIN, J., STEVENSON, H. \& WEI SKILLERN, J. (2006): "Social and commercial entrepreneurship: same, different, or both?", Entrepreneurship Theory and Practice, 30(1), 1-22.

BAGOZZI, R.P. (2010): "Structural equation models are modelling tools with many ambiguities: Comments acknowledging the need for caution and humility in their use", Journal of Consumer Psychology, 20(2), 208-214.

BENTLER, P.M. (2006): EQS Structural Equations Program Manual. Multivariate Software Inc., California.

BELLOSTAS, A.J., LÓPEZ-ARCEIZ, F.J. \& MATEOS, L. (2016): "Social value and economic value in social enterprises: Value creation model of Spanish sheltered workshops", Voluntas, 27(1), 367391.

BINGHAM, J.B., DYER, W.G., SMITH, I. \& ADAMS, G.L. (2011): "A stakeholder identity orientation approach to corporate social performance in family firms", Journal of Business Ethics, 99(4), 565585.

BOSCHEE, J. (2001): "Eight basic principles for nonprofit entrepreneurs", Nonprofit World, 19(4), 15-18.

BOUSSEMART, J.P., SHEN, Z., LELEU, H. \& VALDMANIS, V. (2017): "Performance analysis for three pillars of sustainability", Working Paper (No. 2017-EQM-01).

BRAMMER, S. \& MILLINGTON, A. (2008): "Does it pay to be different? An analysis of the relationship between corporate social and financial performance", Strategic Management Journal, 29(12), 1325-1343. 
BURNS, D.C., SALE, J.T. \& STEPHAN, J.A. (2008): "A better way to gauge profitability", Journal of Accountancy, 206(2), 38.

CASTRILLON, M.A. \& MARES, A.I. (2016): "La empresa familiar, revisión documental", Desarrollo Gerencial, 4(2), 174-214.

CEPES (2011): El impacto socioeconómico de las entidades de economía social. Identificación, medición y valoración de los efectos vinculados a los principios de actuación de las empresas de la economía social, CEPES, España.

CHAD, P. (2013): "Extending the use of market orientation: Transforming a charity into a business", Australasian Marketing Journal, 21(1), 10-16.

CHANG, K.J., CHICHERNEA, D.C. \& HASSABELNABY, H.R. (2014): "On the DuPont analysis in the health care industry", Journal of Accounting and Public Policy, 33(1), 83-103.

CHAVES, R. \& MONZON, J.L. (2012): The social economy in the European Union, CESE/COMM/05/2005, http://www.socialeconomy.eu.org/spip.php?article421.

CLIFFORD, J., MARKEY, K. \& MALPANI, N. (2013): Measuring social impact in social enterprise: The state of thought and practice in the UK, E3M, London.

COMISIÓN EUROPEA [Estrategia UE 2020] (2010): Una estrategia para un crecimiento inteligente, sostenible e integrador (COM(2010) 2020 final), http://ec.europa.eu/europe2020/index_es.htm, [Consulta realizada el día 12 de Febrero de 2016].

COMISIÓN EUROPEA (2011): Social business initiative creating a favorable climate for social enterprises, key stakeholders in the social economy and innovation (COM/2011/0682 final) [Consulta realizada el 30 de Octubre de 2017].

COMISIÓN EUROPEA (2014): Empresas sociales, http://ec.europa.eu/growth/sectors/social-economy/enterprises_es [Consulta realizada el día 21 de Junio de 2017].

COMISIÓN EUROPEA (2014): Métodos propuestos para la medición del impacto social [Consulta realizada el 30 de Octubre de 2017].

CREDIT SUISSE (2010): Funding as a family: Engaging the next generation in family philanthropy (White Paper 02), Credit Suisse Securities, USA.

CUGANESAN, S. (2006): "Reporting Organizational Performance in Managing Human Resources: Intellectual Capital or Stakeholder Perspectives?", Journal of Human Resource Costing \& Accounting, 10(3), 164-88.

DEES, J.G. (2001): The meaning of Social Entrepreneurship, Kauffman Center for Entrepreneurial Leadership, Stanford.

DEFOURNY, J. \& NYSSENS, M. (2008): Social enterprise in Europe: New trends and development (No. 08/1), EMES: Working paper. 
DEFOURNY, J. \& NYSSENS, M. (2012): "El enfoque EMES de empresa social desde una perspectiva comparada", CIRIEC-España, Revista de Economía Pública, Social y Cooperativa, 75, 7-34.

DEFOURNY, J. \& NYSSENS, M. (2013): "Social co-operatives: When social enterprises meet the cooperative tradition", Journal of Entrepreneurial and Organizational Diversity, 2(2), 11-33.

DEFOURNY J., GRØNBJERG, K., MEIJS, L., NYSSENS, M. \& YAMAGUCHI, N. (2016): "Symposium: Comments on Salamonand Sokolowski's Re-conceptualization of the Third Sector", Voluntas, International Journal of Voluntary and Nonprofit Organizations, 27, 1546-1561.

DEL SOL, I. (2015): La empresa familiar en España, Instituto de la Empresa Familiar: Madrid.

DELOITTE (2015): Informe del impacto social de las empresas, Deloitte: Madrid.

DÍAZ-FONCEA, M. \& MARCUELLO, C. (2012): "Social enterprises and social markets: models and new trends", Service business, 6(1), 61-83.

DILLENBURG, S., GREENE, T. \& EREKSON, O.H. (2003): "Approaching socially responsible investment with a comprehensive ratings scheme: Total social impact", Journal of Business Ethics, 43(3), 167-177.

Directorio de Fundaciones de Aragón (2015):

http:/www.aragon.es/estaticos/GobiernoAragon/Departamentos/PoliticaTerritoriallnterior/Areas/

03_Asociaciones_Fundaciones_Colegios_profesionales/03_Fundaciones/directorio\%20fundacio nes\%202012.pdf [Consulta realizada el día 13 de Febrero de 2016].

DONCKELS, R. (1998): "Ondernemen in het familiebedrijf”. En: Scherjon, D.P. \& Thurik, A.R. (Eds.), Handboek ondernemers en adviseurs in het midden en Kleinbedrijf, Kluwer BedrijfsInformatie, Devanter.

DORADO, S. (2006): "Social entrepreneurial ventures: Different values so different process of creation, no?", Journal of Developmental Entrepreneurship, 11(4), 1-24.

EIKENBERRY, A.M. \& KLUVER, J.D. (2004): "The marketization of the nonprofit sector: Civil society at risk?", Public Administration Review, 64(2), 132-140.

EMERSON, J. \& TWERSKY, F. (1996): New social entrepreneurs: The success, challenge and lessons of non-profit enterprise creation, The Roberts Foundation Homeless Economic Fund, San Francisco.

EMERSON, J. (2006): "Moving ahead together: Implications of a blended value framework for the future of social entrepreneurship". In: Nicholls, A. (ed.): Social entrepreneurship: New paradigms of sustainable social change, Oxford University Press, Oxford, 391-406.

ETCHART, N. \& DAVIS, L. (Eds.) (1999): Profits for nonprofits: The NGO Venture Forum, NESsT, San Francisco. 
FAYOLLE, A. \& MATLAY, H. (Eds.) (2010): Handbook of research on social entrepreneurship, Edward Elgar Publishing, USA.

FELÍCIO, J.A., MARTINS GONÇALVES, H. \& DA CONCEIÇÃO GONÇALVES, V. (2013): "Social value and organizational performance in non-profit social organizations: social entrepreneurship, leadership, and socioeconomic context effects", Journal of Business Research, 66(10), 2139-2146.

FELIU, N. \& BOTERO, I.C. (2015): "Philanthropy in family enterprises: A review of literature", Family Business Review, (Forthcoming).

GALERA, G. \& BORZAGA, C. (2009): "Social enterprise: An international overview of its conceptual evolution and legal implementation", Social Enterprise Journal, 5(3), 210-228.

GEORGE, D. \& MALLERY, P. (2003): SPSS for Windows step by step: A Simple guide and reference. 11.0 Update, Allyn \& Bacon, Boston.

GERSICK, K. \& FELIU, N. (2014): "Governing the family enterprise: Practices, performance, and research". En: Melin, L., Nordqvist, M. \& P. Sharma (Eds.), SAGE handbook of family business, SAGE, Thousand Oaks CA, 196-225.

GHEORGHE, S. (2013): "The analysis of profitability indicators", Annals-Economy Series, 4, 132-138.

GÓMEZ, F. (2008): "Responsabilidad Social Corporativa y Performance financiera: treinta y cinco años de investigación empírica en busca de un consenso", Principios, 11, 5-22.

GRUPO EUROPEO DE EMPRESAS FAMILIARES (2008): ¿Qué es la empresa familiar?, ADEFAM, [Consultado el 15 de abril de 2016].

GUZMAN VÁSQUEZ, A.G. \& TRUJILLO DÁVILA, M.A. (2008): "Emprendimiento social-Revisión de literatura", Estudios Gerenciales, 24(109), 105-125.

HAHN, T. \& FIGGE, F. (2011): "Beyond the bounded instrumentality in current corporate sustainability research: Toward an inclusive notion of profitability", Journal of Business Ethics, 104(3), 325345.

HALL, A., MELIN, L. \& NORDQVIST, M. (2001): "Entrepreneurship as radical change in the family business: Exploring the role of cultural patterns", Family Business Review, 14(3), 193-208.

HARDING, R. (2004): "Social enterprise: the new economic engine?", Business Strategy Review, 15(4), 39-43.

HAYDUK, L.A. \& LITTVAY, L. (2012): "Should researchers use single indicators, best indicators, or multiple indicators in structural equation models?", BMC Medical Research Methodology, 12(1), 12-159.

HERCIU, M., OGREAN, C. \& BELASCU, L. (2011): "A DuPont analysis of the 20 most profitable companies in the world", Group, 13(1), 18-93. 
HULGÅRD, L. (2010): Discourses of social entrepreneurship-Variations of the same theme?, (No 10/01), SE Field Working Paper.

IBRAHIM, A.B., SOUFANI, K. \& LAM, J. (2001): "A study of succession in a family firm", Family Business Review, 14(3), 245-258.

KAPLUN, C. (2012): La familia en la empresa (Parte I), Universidad de Monterrey, México.

KERLIN, J.A. (2006): "Social enterprise in the United States and Europe: Understanding and learning from the differences", Voluntas, International Journal of Voluntary and Nonprofit Organizations, 17(3), 247-263.

LATTUADA, M., NOGUEIRA, M.E. \& URCOLA, M. (2015): "Las formas asociativas de la agricultura familiar en el desarrollo rural argentino de las últimas décadas (1990-2014)", CIRIEC-España, Revista de Economía Pública, Social y Cooperativa, 84, 195-228.

LOCKETT, A., MOON, J. \& VISSER, W. (2006): "Corporate Social Responsibility in management research: Focus, nature, salience and sources of influence", Journal of Management Studies, 43(1), 115-135.

LÓPEZ-CÓZAR, C., PRIEDE-BERGAMINI, T. \& HILLIARD, I. (2014): "Family and non-family business differences in corporate social responsibility approaches", ASEAN Journal of Management \& Innovation, 1(2), 1-12.

LÓPEZ ARCEIZ, F.J., BELLOSTAS, A.J. \& RIVERA, M.P. (2016). "The effects of resources on social activity and economic performance in Social Economy organizations", Nonprofit Management and Leadership, 26(4), 499-511.

MAKNI, R., FRANCOEUR, C. \& BELLAVANCE, F. (2009): "Causality between corporate social performance and financial performance: Evidence from Canadian firms", Journal of Business Ethics, 89(3), 409-422.

MAIR, J. \& MARTI, I. (2006): "Social entrepreneurship research: A source of explanation, prediction, and delight", Journal of World Business, 41(1), 36-44.

MANO, R. (2014): "Networking Modes and Performance in Israel's Nonprofit Organizations", Nonprofit Management and Leadership, 24 (4), 429-44.

MANO, R. (2015): "Funding Allocations in Israel: An Empirical Assessment of the New Philanthropy Approach", Voluntas, International Journal of Voluntary and Nonprofit Organizations, 26(5), 21302145.

MARGOLIS, J.D., ELFENBEIN, H.A. \& WALSH, J.P. (2007): "Does it pay to be good? A meta-analysis and redirection of research on the relationship between corporate social and financial performance", Ann Arbor, 1001, 48109-1234. 
MARTÍN BAILÓN, S., BELLOSTAS PEREZGRUESO, A.J. \& LÓPEZ-ARCEIZ, F.J. (2017): “¿Son los voluntarios un recurso sustitutivo de los trabajadores? una aproximación desde la economía social aragonesa?", REVESCO, Revista de Estudios Cooperativos, 123, 143-173.

MCWILLIAMS, A. \& SIEGEL, D. (2000): "Corporate Social Responsibility and financial performance: Correlation or misspecifications?", Strategic Management Journal, 21, 603-609.

MCWILLIAMS, A., SIEGEL, D. \& TEOH, S.H. (1999): "Issues in the use of the event study methodology: A critical analysis of Corporate Social Responsibility studies", Organizational Research Methods, 2, 340-365.

MEDINA GIACOMOZZI, A., GALLEGOS MUÑOZ, C. \& LARA HADI, P. (2008): "Motivación y satisfacción de los trabajadores y su influencia en la creación de valor económico en la empresa", Revista de Administraçao Pública, 42(6), 1213-1230.

NOGALES MURIEL, R., BORZAGA, C. \& GALERA, G. (2008): Social Enterprise: A New Model for Poverty Reduction and Employment Generation, Bratislava - Lieja, UNDP Bratislava Regional Center/EMES.

NOGALES MURIEL, R. (2007): "Aparición y evolución de la empresa social en Europa", Economía social. 39, 44-48.

NOGALES MURIEL, R. (2017): "La empresa social en Europa y España: evolución, relevancia y desafíos", Revista Española del Tercer Sector, 35, 117-140.

O'BOYLE, E.H., RUTHERFORD, M.W. \& POLLACK, J.M. (2010): "Examining the relation between ethical focus and financial performance in family firms: An exploratory study", Family Business Review, 23(4), 310-326.

ORLITZKY, M., SCHMIDT, F. \& RYNES, S. (2003): "Corporate social and financial performance: a meta-Analysis", Organization Studies, 24, 403-441.

ORTAS, E. \& MONEVA, J.M. (2010): "Corporate environmental and financial performance: a multivariate approach", Industrial Management \& Data Systems, 110(2), 193-210.

PEARCE, J. \& KAY, A. (2003): Social enterprise in anytown, Calouste Gulbenkian Foundation, UK.

PEREDO, A.M. \& MCLEAN, M. (2006): "Social entrepreneurship: A critical review of the concept", Journal of World Business, 41(1), 56-65.

PIRSON, M. (2011): Social entrepreneurs as the paragons of shared value creation? A critical perspective, Fordham University Schools of Business Working Paper.

PIRVU, D., UNGUREANU, E. \& HAGIU, A. (2009): "Evaluation of the need for development of social enterprises. Case study in the Arge County", Revista de Cercetare i Interven ie Social, 27, 51-65.

POLONSKY, M. \& GRAU, S. (2011): "Assessing the social impact of charitable organizations-four alternative approaches", International journal of nonprofit and voluntary sector marketing, 16(2), 195211. 
POLONSKY, M., GRAU, S. \& MCDONALD, S. (2016): "Perspectives on social impact measurement and non-profit organisations", Marketing Intelligence \& Planning, 34(1), 80-98.

PORTER, M.E. \& KRAMER, M.R. (2011): "Creating shared value. How to reinvent capitalism- and unleash wave of innovation and growth", Harvard Business Review, January-February, 1-17.

POYATOS LEÓN, J.A. (2015): Análisis de la relación causal de la responsabilidad social corporativa y la performance financiera de las empresas (Doctoral dissertation), Universidad Politécnica de Valencia, Valencia.

QUINTÃO, C. (2007): "Empresas de inserción y empresas sociales en Europa", CIRIEC-España, Revista de Economía Pública, Social y Cooperativa, 59, 33-60.

RED DE CÁTEDRAS DE EMPRESA FAMILIAR (2015): http://www.iefamiliar.com/web/es/catedras.html [Consulta realizada el día 13 de Febrero de 2016].

RETOLAZA, J.L. \& SAN-JOSÉ, L. (2016): "Contabilidad social para la sostenibilidad: modelo y aplicación", Revista de Contabilidad y Dirección, 23, 159-178.

REY-GARCIA, M. (2012): Beyond corporate social responsibility: Foundations and global retailers, DOCFRADIS Working Papers, n. 1206.

REY-GARCIA, M. \& PUIG-RAPOSO, N. (2013): "Globalisation and the organisation of family philanthropy: A case of isomorphism?", Business History, 55(6), 1019-1046.

SALAMON, L.M. \& SOKOLOWSKI, S.W. (2016): "Beyond Nonprofits: Re-conceptualizing the Third Sector". VOLUNTAS, International Journal of Voluntary and Nonprofit Organizations, 27, 15151545.

SANTOS, J. (2015): Tipos de subvenciones y ayudas existentes, El Economista, Madrid.

SEKN (2006): Effective management in social enterprise: Lessons from business and civil society organizations in Iberoamerica, David Rockefeller Center, Boston.

SHARMA, P. (2004): "An overview of the field of family business studies: Current status and directions for the future", Family Business Review, 17(1), 1-36.

SUD, M., VANSANDT, C.V. \& BAUGOUS, A.M. (2009): "Social entrepreneurship: The role of institutions", Journal of Business Ethics, 85(1), 201-216.

THOMPSON, J. \& DOHERTY, B. (2006): "The diverse world of social enterprise: A collection of social enterprise stories", International Journal of Social Economics, 33(5/6), 361-375.

TRAVAGLINI, C., BANDINI, F. \& MANCINONE. K. (2010): Social enterprise in Europe: Governance models. Analysis of governance models in social enterprises through a comparative study of the legislation of eleven countries, AICCON Working Papers. 
TSANG, E.W. (2002): "Learning from overseas venturing experience: The case of Chinese family businesses", Journal of Business Venturing, 17(1), 21-40.

VALLEJO, M.C. (2005): "Cuando definir es una necesidad. Una propuesta integradora y operativa del concepto de empresa familiar", Investigaciones europeas de dirección y economía de la empresa, 11(3), 151-171.

VAN BEURDEN, P. \& GÖSSLING, T. (2008): "The worth of values-a literature review on the relation between corporate social and financial performance", Journal of Business Ethics, 82(2), 407-424.

WADDOCK, S. \& GRAVES, S. (1997): "The corporate social performance - financial performance link", Strategic Management Journal, 18, 303-319.

WU, M.L. (2006): "Corporate social performance, corporate financial performance, and firm size: A meta-analysis", Journal of American Academy of Business, 8(1), 163-171.

YOUNG, D.R. \& SALAMON, L.M. (2002): "Commercialization, social ventures, and for-profit competition". En: Salamon, L.M. (Ed.), The state of nonprofit America, Brookings, Washington DC, 423446.

YUNUS, M. (2010): Building Social Business. Capitalism that can serve humanity's most pressing needs, Public Affairs, New York. 


\section{Anexo I. Relación entre valor económico y social por año y tipo de entidad}

\begin{tabular}{|c|c|c|c|c|c|c|c|c|c|c|c|c|}
\hline & \multicolumn{4}{|c|}{$\mathrm{FL}$} & \multicolumn{4}{|c|}{ FNL } & \multicolumn{2}{|c|}{$x^{2}$} \\
\hline & & & M & Rot & EG & ES & M & Rot & $E G$ & ES & M & Rot \\
\hline \multirow[t]{5}{*}{2009} & M & Margen & & & & & & & & & & \\
\hline & Rot & Rotación & 0.000 & & & & -0.051 & & & & & \\
\hline & EG & Empleabilidad & -0.005 & 0.000 & & & -0.085 & 0.047 & & & - & - \\
\hline & ES & Empleabilidad subvencionada & $0.037^{\star \star}$ & -0.003 & 0.026 & & 0.050 & -0.035 & -0.015 & & - & - \\
\hline & ENS & Empleabilidad no subvencionada & 0.001 & 0.006 & $-0.092^{* * *}$ & $0.283^{\star \star \star *}$ & -0.047 & 0.003 & 0.056 & $-0.665^{* * *}$ & - & - \\
\hline \multirow[t]{5}{*}{2010} & M & Margen & & & & & & & & & & \\
\hline & Rot & Rotación & 0.000 & & & & 0.092 & & & & & \\
\hline & EG & Empleabilidad & -0.001 & 0.000 & & & $-0.347^{\star \star \star}$ & 0.067 & & & - & * \\
\hline & ES & Empleabilidad subvencionada & $-0.080^{* * *}$ & -0.001 & -0.009 & & 0.107 & -0.004 & 0.033 & & - & - \\
\hline & ENS & Empleabilidad no subvencionada & 0.002 & 0.000 & -0.001 & $0.437^{\star \star *}$ & 0.031 & 0.001 & -0.059 & $-0.861^{* * *}$ & - & $\cdot$ \\
\hline \multirow[t]{5}{*}{2011} & M & Margen & & & & & & & & & & \\
\hline & Rot & Rotación & -0.002 & & & & -0.132 & & & & & \\
\hline & EG & Empleabilidad & -0.029 & 0.003 & & & -0.140 & -0.059 & & & - & - \\
\hline & ES & Empleabilidad subvencionada & -0.009 & -0.002 & $-0.036^{*}$ & & -0.046 & -0.004 & 0.028 & & - & - \\
\hline & ENS & Empleabilidad no subvencionada & -0.003 & 0.013 & -0.011 & $0.265^{\star * \star}$ & 0.045 & 0.006 & -0.043 & $-0.976^{* * *}$ & - & $\cdot$ \\
\hline \multirow[t]{5}{*}{2012} & M & Margen & & & & & & & & & & \\
\hline & Rot & Rotación & 0.000 & & & & 0.024 & & & & & \\
\hline & EG & Empleabilidad & -0.010 & 0.002 & & & -0.054 & 0.028 & & & - & - \\
\hline & ES & Empleabilidad subvencionada & 0.008 & -0.001 & -0.028 & & 0.082 & $-0.294^{* * *}$ & 0.023 & & - & - \\
\hline & ENS & Empleabilidad no subvencionada & -0.001 & -0.007 & -0.013 & $0.337^{\star \star \star}$ & -0.078 & $0.291^{* * *}$ & -0.027 & $-0.999^{* * *}$ & - & - \\
\hline \multirow[t]{5}{*}{2013} & M & Margen & & & & & & & & & & \\
\hline & Rot & Rotación & 0.001 & & & & -0.029 & & & & & \\
\hline & EG & Empleabilidad & -0.004 & 0.000 & & & -0.026 & 0.031 & & & - & $\cdot$ \\
\hline & ES & Empleabilidad subvencionada & -0.005 & 0.000 & -0.015 & & $-0.253^{\star *}$ & -0.092 & -0.044 & & - & * \\
\hline & ENS & Empleabilidad no subvencionada & 0.000 & 0.005 & -0.017 & -0.024 & 0.032 & 0.046 & $0.617^{\star \star \star}$ & $-0.470^{\star * \star}$ & - & - \\
\hline
\end{tabular}

${ }^{* * *}$ pvalor<0.01; ** pvalor<0.05; " pvalor<0.10; FL: Forma lucrativa (Modelos 1 y 2); FNL: Forma no lucrativa (modelos 3 y 4 ) 


\section{Anexo II. Relación entre valor económico y social por año y tipo de actividad}

\begin{tabular}{|c|c|c|c|c|c|c|c|c|c|c|c|c|c|c|}
\hline & & & \multicolumn{4}{|c|}{ AGRI } & \multicolumn{4}{|c|}{ INDT } & \multicolumn{4}{|c|}{ CULT } \\
\hline & & & M & Rot & $E G$ & ES & M & Rot & EG & ES & M & Rot & $E G$ & ES \\
\hline \multirow[t]{5}{*}{2009} & M & Margen & & & & & & & & & & & & \\
\hline & Rot & Rotación & 0,058 & & & & $-0,003$ & & & & $-0,004$ & & & \\
\hline & EG & Empleab & $-0,035$ & 0,009 & & & 0,005 & $-0,010$ & & & $-0,050$ & 0,001 & & \\
\hline & ES & Empleab subv & $-0,018$ & 0,023 & $0,474^{* \star *}$ & & $0,113^{* \star *}$ & 0,026 & 0,000 & & $-0,067$ & $-0,005$ & 0,095 & \\
\hline & ENS & Empleab no subv & 0,024 & 0,009 & $-0,607^{* \star *}$ & $0,223^{\star \star *}$ & 0,003 & $-0,007$ & $0,056^{*}$ & $0,455^{\star \star *}$ & 0,075 & 0,030 & $-0,061$ & $-0,466^{* * *}$ \\
\hline \multirow[t]{5}{*}{2010} & M & Margen & & & & & & & & & & & & \\
\hline & Rot & Rotación & -0.035 & & & & 0,001 & & & & 0,001 & & & \\
\hline & EG & Empleab & 0,068 & 0,019 & & & 0,004 & 0,002 & & & $-0,044$ & 0,064 & & \\
\hline & ES & Empleab subv & $-0,202^{* *}$ & 0,029 & 0,027 & & $-0,210^{* * *}$ & $-0,002$ & $-0,011$ & & 0,012 & 0,003 & 0,017 & \\
\hline & ENS & Empleab no subv & 0,007 & 0,023 & $-0,002$ & $0,552^{\star \star \star}$ & 0,009 & $-0,002$ & $-0,012$ & $0,633^{* * *}$ & 0,009 & $-0,006$ & $-0,062$ & $-0,403^{* * *}$ \\
\hline \multirow[t]{5}{*}{2011} & M & Margen & & & & & & & & & & & & \\
\hline & Rot & Rotación & 0,000 & & & & $-0,001$ & & & & $-0,038$ & & & \\
\hline & EG & Empleab & $-0,076$ & 0,009 & & & $-0,094^{\star \star \star}$ & 0,014 & & & $-0,791^{* * \star}$ & $-0,009$ & & \\
\hline & ES & Empleab subv & $-0,004$ & 0,002 & $-0,081$ & & $-0,006$ & 0,006 & 0,013 & & $-0,019$ & 0,003 & 0,018 & \\
\hline & ENS & Empleab no subv & 0,005 & 0,035 & $-0,022$ & $0,556^{\star \star \star}$ & 0,001 & 0,014 & 0,001 & $0,166^{\star \star \star}$ & 0,004 & 0,001 & $-0,020$ & $-0,961^{* * *}$ \\
\hline \multirow[t]{5}{*}{2012} & M & Margen & & & & & & & & & & & & \\
\hline & Rot & Rotación & 0,025 & & & & 0,003 & & & & $-0,052$ & & & \\
\hline & EG & Empleab & $-0,013$ & $-0,005$ & & & $-0,003$ & 0,002 & & & $-0,192^{* *}$ & 0,069 & & \\
\hline & ES & Empleab & 0,026 & 0,002 & $-0,028$ & & 0,045 & $-0,006$ & $-0,011$ & & 0,009 & $-0,278^{\star \star *}$ & 0,000 & \\
\hline & ENS & Empleab no subv & 0,014 & 0,000 & $-0,014$ & $0,660^{* * *}$ & $-0,001$ & $-0,007$ & $-0,049^{*}$ & $0,232^{* \star *}$ & $-0,009$ & $0,276^{* * *}$ & $-0,003$ & $-0,998^{* * *}$ \\
\hline \multirow[t]{5}{*}{2013} & M & Margen & & & & & & & & & & & & \\
\hline & Rot & Rotación & 0,006 & & & & 0,003 & & & & $-0,066$ & & & \\
\hline & $E G$ & Empleab & $-0,171^{* *}$ & $-0,008$ & & & 0,014 & 0,022 & & & $-0,091$ & 0,042 & & \\
\hline & ES & Empleab & 0,104 & 0,027 & $-0,391^{\star * *}$ & & $-0,002$ & 0,000 & $-0,014$ & & $-0,171^{* *}$ & $-0,055$ & $-0,001$ & \\
\hline & ENS & Empleab & 0,060 & 0,066 & $-0,160^{*}$ & 0,112 & 0,001 & 0,004 & 0,043 & $0,216^{* * *}$ & $-0,205^{\star \star \star}$ & 0,017 & 0,040 & $0,687^{* * *}$ \\
\hline
\end{tabular}

${ }^{* * *}$ pvalor<0.01; **pvalor<0.05; " pvalor<0.10; AGRI: Agricultura INDT: Industria de transformación EDU: Educación, cultura y artes 


\section{Anexo II. Relación entre valor económico y social por año y tipo de actividad (continuación)}

\begin{tabular}{|c|c|c|c|c|c|c|c|c|c|c|c|c|c|c|c|c|}
\hline & & & \multicolumn{4}{|c|}{ SERV } & \multicolumn{4}{|c|}{ SAN } & \multicolumn{4}{|c|}{ COME } & \multicolumn{2}{|c|}{$x^{2}$} \\
\hline & & & M & Rot & $E G$ & ES & M & Rot & $E G$ & ES & M & Rot & $E G$ & ES & & Rot \\
\hline \multirow[t]{5}{*}{2009} & M & Margen & & & & & & & & & & & & & & \\
\hline & Rot & Rotación & 0,004 & & & & $-0,010$ & & & & 0,000 & & & & & \\
\hline & $E G$ & Empleab & $-0,017$ & 0,000 & & & $-0,320^{*}$ & $-0,228$ & & & $-0,007$ & 0,000 & & & * & - \\
\hline & ES & Empleab subv & $-0,005$ & 0,001 & $-0,181^{* * *}$ & & 0,039 & 0,030 & $-0,134$ & & 0,000 & $-0,009$ & $-0,007$ & & - & - \\
\hline & ENS & Empleab no subv & 0,000 & 0,026 & $-0,012$ & $0,265^{\star * \star}$ & 0,043 & 0,023 & $-0,137$ & $0,995^{* * \star}$ & 0,000 & 0,008 & 0,004 & $0,190^{\star * \star}$ & - & - \\
\hline \multirow[t]{5}{*}{2010} & M & Margen & & & & & & & & & & & & & & \\
\hline & Rot & Rotación & $-0,005$ & & & & 0,129 & & & & 0,000 & & & & & \\
\hline & $E G$ & Empleab & 0,004 & 0,002 & & & $-0,435^{\star \star \star}$ & $-0,358^{* *}$ & & & $-0,003$ & 0,000 & & & - & * \\
\hline & ES & Empleab subv & $-0,026$ & 0,007 & $-0,228^{* * *}$ & & $-0,004$ & 0,024 & $-0,066$ & & $-0,010$ & $-0,010$ & $-0,003$ & & - & - \\
\hline & ENS & Empleab no subv & 0,022 & 0,009 & $-0,046$ & $0,777^{\star * *}$ & $-0,006$ & 0,013 & $-0,069$ & $0,995^{* *}$ & 0,000 & 0,012 & 0,002 & $0,176^{\star \star \star}$ & - & - \\
\hline \multirow[t]{5}{*}{2011} & M & Margen & & & & & & & & & & & & & & \\
\hline & Rot & Rotación & $-0,003$ & & & & $-0,121$ & & & & 0,003 & & & & & \\
\hline & $E G$ & Empleab & $-0,003$ & 0,004 & & & $-0,238$ & $-0,170$ & & & $-0,005$ & $-0,001$ & & & - & - \\
\hline & ES & Empleab subv & $-0,017$ & $-0,013$ & $-0,246^{* * *}$ & & $-0,052$ & 0,070 & 0,057 & & $-0,010$ & 0,000 & 0,003 & & - & - \\
\hline & ENS & Empleab no subv & $-0,012$ & 0,021 & $-0,055$ & $0,542^{* * *}$ & 0,000 & 0,011 & $-0,028$ & $0,687^{* \star *}$ & $-0,006$ & 0,015 & 0,005 & $0,308^{\star \star *}$ & - & - \\
\hline \multirow[t]{5}{*}{2012} & M & Margen & & & & & & & & & & & & & & \\
\hline & Rot & Rotación & $-0,010$ & & & & $-0,111$ & & & & $-0,001$ & & & & & \\
\hline & $E G$ & Empleab & $-0,028$ & 0,002 & & & $-0,133$ & 0,031 & & & $-0,009$ & 0,001 & & & - & - \\
\hline & ES & Empleab & $-0,016$ & 0,009 & $-0,126^{* * *}$ & & $-0,257$ & $-0,070$ & $-0,018$ & & $-0,009$ & 0,009 & $-0,005$ & & - & - \\
\hline & ENS & Empleab no subv & 0,002 & $-0,003$ & $-0,064$ & $0,870^{* * *}$ & $-0,261$ & $-0,067$ & $-0,026$ & $0,985^{\star \star *}$ & 0,000 & $-0,059^{*}$ & $0,057^{\star}$ & $0,210^{\star \star \star}$ & - & - \\
\hline \multirow[t]{5}{*}{2013} & $M$ & Margen & & & & & & & & & & & & & & \\
\hline & Rot & Rotación & $-0,003$ & & & & $-0,131$ & & & & 0,001 & & & & & \\
\hline & $E G$ & Empleab & $-0,006$ & 0,022 & & & $-0,036$ & $-0,015$ & & & $-0,009$ & $-0,024$ & & & * & - \\
\hline & ES & Empleab & $-0,068$ & $-0,001$ & $0,088^{\star *}$ & & $-0,202$ & $-0,108$ & $-0,328^{*}$ & & 0,003 & 0,000 & $-0,025$ & & - & . \\
\hline & ENS & Empleab & 0,009 & 0,000 & $-0,012$ & $0,797^{\star * *}$ & $-0,137$ & $-0,087$ & $-0,346^{* *}$ & $0,819^{* * *}$ & $-0,002$ & 0,004 & $-0,067^{\star *}$ & $-0,363^{* * *}$ & - & . \\
\hline
\end{tabular}

***pvalor<0.01; * ${ }^{*}$ pvalor<0.05; " pvalor<0.10; SERV: Servicios profesionales SAN: Sanidad y servicios sociales COME: Comercio y hostelería. 
